\title{
REPRESENTATIONS OF THE GENERAL SYMMETRIC GROUP AS LINEAR GROUPS IN FINITE AND INFINITE FIELDS*
}

\author{
BY

\section{LEONARD EUGENE DICKSON}

1. In a series of articles in the Berliner Berichte, beginning in 1896, Frobenius has developed an elaborate theory of group-characters and applied it to the representation of a given finite group $G$ as a non-modular linear group. Later, BuRnsIDE $\nmid$ approached the subject from the standpoint of continuous groups. The writer has shown $\ddagger$ that the method employed by Bursside may be replaced by one involving only purely rational processes and bence leading to results valid for a general field. The last treatment, however, expressly excludes the case in which the field has a modulus which divides the order of $G$. The exclusion of this case is not merely a matter of convenience, nor merely a limitation due to the particular method of treatment; indeed, $\S$ the properties of the group-determinant differ essentially from those holding when the modulus does not divide the order of $G . \|$ Thus when $G$ is of order $q$ !, the general theory gives no information as to the representations in a field having a modulus $\leqq q$, whereas the case of a small modulus is the most important one for the applications.

The present paper investigates the linear homogeneous groups on $m$ variables, with coefficients in a field $\boldsymbol{F}$, which are simply isomorphic with the symmetric group on $q$ letters. The treatment is elementary and entirely independent of the papers cited above; in particular, the investigation is made for all moduli without exception. The principal result is the determination of the minimum value of the number of variables. It is shown that $m \geqq q-1$ or $m \geqq q-2$, according as $F$ has not or has a modulus $p$ which divides $q$ (\$\$ 8-21). There

* Presented to the Society (Chicago), March 30, 1907. Received for publication April 17, 1907:

† Proceedings of the London Matbematical Sooiety, vol. 29 (1898), pp. 207-224, 546-565; vol. 35 (1902), pp. 206-220.

† These Transactions, rol. 3 (1902), pp. 285-301.

8 Dickson, Proceedings of the London Mathematioal Sooiety, vol. 35 (1902), p. 68.

$\|$ Since the present paper was written, the writer has obtained some general results on the ontstanding case in which the modulus divides the order of $G$, these Transactions, vol. 8 (1907), pp. 389-398; Bulletin of the Amerioan Mathematioal Society, vol. 13 (1907), pp. 477-488. 
is a representation on $q-1$ variables, except in the trivial case $q=p=2(\S 2)$; while for any field having a modulus which divides there is a representation on $q-2$ variables, except in the special cases $q \leqq 4(\S 4)$.

The determination of the possible representations is made for a case considerably more general than the special one found to be sufficient in establishing the minimum value of $m$ (cf. $\$ 11$ ). We thereby gain a better insight into the nature of the general problem. The question of the equivalence, under linear transformation, of the resulting linear groups is treated in $\$ \S 22-24$.

The most essential point in the proof leading to a minimum for $m$ may be seen in a typical case in $\S 13$. After the preliminary normalizations, the transformations involve two sets of parameters. With these we form two rectangular matrices $A, B$. Then the conditions on the parameters are equivalent to the condition that the product $A B$ shall equal a known matrix $M$. The representation on $m$ variables is thus possible if and only if the elements of the two matrices $A$ and $B$ can be determined in the field so that $A B=M$. The problem is thus reduced to a simple question of determinants.

2. The symmetric group $S_{q !}$ on $q$ letters is isomorphic with the group of the transformations

$$
x_{i}^{\prime}=x_{a_{i}} \quad\left(a^{\prime} \text { s a permutation of } 1, \cdots, q\right) .
$$

In place of $x_{q}$ we introduce the new variable

$$
\omega=x_{1}+\cdots+x_{q},
$$

and then suppress $\omega$. According as $a_{q}=q$ or $a_{t}=q(t<q)$, we get

$$
\text { (4) } x_{t}^{\prime}=-\sum_{j=1}^{q-1} x_{j}, \quad x_{i}^{\prime}=x_{a_{i}}\left[\text { the } a_{i}(i \neq t) \text { a permutation of } 1, \cdots, q-1 \text { with } a_{q}\right. \text { omitted ]. }
$$

$$
\left.x_{i}^{\prime}=x_{a_{i}} \quad \text { (a's a permutation of } 1, \cdots, q-1\right),
$$

Except when $q=2$ and the coefficients are taken modulo 2, (4) is not the identity $I$, and the isomorphism is simple. Henceforth we shall take $q>2$.

3. The transformations (1) leave invariant (2) and

$$
\omega_{2}=\sum x_{i} x_{j}, \quad \omega_{3}=\sum x_{i} x_{j} x_{k} \quad(i, j, k=1, \cdots, q ; i<j<k \cdots) .
$$

Eliminating $x_{q}$ from $\omega_{2}$ by means of (2), we get

where

$$
\omega_{2}=\omega \sum_{i=1}^{q-1} x_{i}-\phi_{q-1}
$$

$$
\phi_{q-1}=\sum x_{i}^{2}+\sum x_{i} x_{j} \quad(i, j=1, \cdots, q-1 ; i<j) .
$$

Hence $\phi$ is an invariant (in fact, the only quadratic invariant) of the group of 
the transformations (3), (4). For the new variables

we have

$$
y_{q-1}=x_{q-1}, \quad y_{i}=x_{i}-x_{q-1} \quad(i=1, \cdots, q-2),
$$

$$
\phi_{q-1}(x)=\phi_{q-2}(y)+q y_{q-1} \sum_{i=1}^{q-2} y_{i}+\frac{1}{2} q(q-1) y_{q-1}^{2} \text {. }
$$

We assume that the coefficients of our transformations and invariants may be reduced modulo $p$, where $p$ is any fixed divisor of $q$. If $p$ is odd, or if both $p$ and $q / p$ are even, the second member of $(8)$ reduces to $\phi_{q-2}(y)$. The possibility of this reduction of the number of variables follows directly from the fact that the discriminant of (6) equals $q$.

4. We next discuss the nature of transformations (3), (4), when expressed on the variables (7). We show that if the coefficients be reduced modulo $p$, a divisor of $q$, then $y_{k}^{\prime}(k<q-1)$ is a linear function of $y_{1}, \cdots, y_{q-2}$. Now $y_{k}^{\prime}=x_{k}^{\prime}-x_{q-1}^{\prime}$. For (3), this equals $x_{a_{k}}-x_{a_{q-1}}$ and hence, by (7), equals a function of. the $y_{i}(i<q-1)$. A like result holds for (4) if $k$ and $q-1$ are both distinct from $t$. If $k=t$ or if $q-1=t, y_{k}^{\prime}$ involves $q x$ 's with like sign and hence equals a function of the $y$ 's in which the coefficient of $y_{q-1}$ is $\pm q$. Hence $S_{q !}$ is simply or multiply isomorphic with a linear homogeneous group $L_{q-2, p}$ on $q-2$ variables modulo $p$. The substitutions of $S$ which correspond to the identity in $L$ form an invariant subgroup $J$ of $S$. For $q>4, J$ is the identity or else it contains all even substitutions on the $q$ letters. But for $q>4,\left[x_{1} x_{2} x_{3}\right] \sim\left[y_{1} y_{2} y_{3}\right]$, so that the isomorphism is simple. For $q=3$,

$$
\left[x_{1} x_{2} x_{3}\right] \sim I, \quad\left[x_{1} x_{2}\right] \sim y_{1}^{\prime}=-y_{1},
$$

so that $L_{1,3}$ is of order 2 , and the isomorphism is $(3,1)$. For $q=4$,

$$
\begin{aligned}
& {\left[x_{1} x_{3}\right]\left[x_{2} x_{1}\right] \sim y_{1}^{\prime}=-y_{1}, \quad y_{2}^{\prime}=-2 y_{1}-y_{2} ;} \\
& {\left[x_{1} x_{4}\right]\left[x_{2} x_{3}\right] \sim y_{1}^{\prime}=-y_{1}-2 y_{2}, \quad y_{2}^{\prime}=-y_{2} ;} \\
& {\left[x_{1} x_{2}\right] \sim\left[y_{1} y_{2}\right], \quad\left[x_{1} x_{3}\right] \sim y_{1}^{\prime}=-y_{1}, \quad y_{2}^{\prime}=-y_{1}+y_{2} .}
\end{aligned}
$$

Hence $S_{4 !}$ is simply isomorphic with $L_{2,4}$, but has $(4,1)$ isomorphism with $L_{2,2}$. Moreover, $S_{4 !}$ cannot be represented as a binary group in a finite or infinite field having modulus 2 . Indeed, the transformations of period 2 are conjugate with $\left(\begin{array}{ll}1 & 0 \\ 1 & 1\end{array}\right)$, which is commutative only with $\left(\begin{array}{ll}a & 0 \\ c & a\end{array}\right)$. The square of the latter is $\left(\begin{array}{cc}a^{2} & 0 \\ 0 & a^{2}\end{array}\right)$. But $a^{4}=1$ requires $a=1$ in any field with modulus 2. Hence there is no binary transformation of period 4 commutative with $\left(\begin{array}{ll}1 & 0 \\ 1 & 1\end{array}\right)$, whereas $S_{4 \text { ! }}$ has a cyclic subgroup of order 4 .

Theorem. For $q>4$, the symmetric group on $q$ letters is simply isomorphic with a linear homogeneous group on $q-2$ variables with integral coefficients taken modulo $p$, where $p$ is any integral divisor $>1$ of $q$. For 
$q=4$, the result holds if $p=4$, but not if $p=2$. The case $q=3$ is exceptional since a unary homogeneous group is commutative.

5.* If $p$ is an odd prime dividing $q$, the group $L_{q-2, p}$ has $(\S 3)$ the invariant $\phi_{q-2}(y)$ of discriminant $q-1 \equiv-1(\bmod p)$. Hence the invariant can be reduced by a linear transformation with integral coefficients to

$$
Y_{\epsilon} \equiv y_{1}^{2}+\cdots+y_{q-3}^{2}+\epsilon y_{q-2}^{2}, \quad\left(\frac{\epsilon}{p}\right)=\left(\frac{-1}{p}\right)\left(\frac{2}{p}\right)^{q-2} .
$$

According as $\epsilon$ is a square or a not-square in a field $F$, the group defined by the invariant $Y_{e}$ is called a first or a second orthogonal group in $F$. In particular, for the $G F\left[p^{n}\right]$ the group is a first or second orthogonal group according as

$$
\left(\frac{-1}{p}\right)^{n}\left(\frac{2}{p}\right)^{n(q-2)}=+1 \text { or }-1 \text {. }
$$

Next let $p=2$. The quadratic form

$$
Q_{2 m}=\sum \xi_{i}^{2}+\sum \xi_{i} \xi_{j} \quad(i, j=1, \cdots, 2 m ; i<j)
$$

can be reduced by a linear transformation with integral coefficients to

$$
f_{c} \doteq \xi_{1} \xi_{2}+\cdots+\xi_{2 m-1} \xi_{2 m}+c\left(\xi_{1}^{2}+\xi_{2}^{2}\right),
$$

where $c \equiv 0$ or $1(\bmod 2)$ according as 2 is a quadratic residue or non-residue of the discriminant $2 m+1$ of $Q_{2 m}$. For the $G F\left[2^{n}\right], f_{1}$ can be reduced to $f_{0}$ if and only if $n$ be even. The group defined by the invariant $f_{0}$ is called the first hypoabelian group; that defined by $f_{1}$ with $\xi_{1} \xi_{2}+\xi_{1}^{2}+\xi_{2}^{2}$ irreducible is called the second hypoabelian group. Hence the group defined by the invariant $Q_{2 m}$ in the $G F\left[2^{n}\right]$ can be transformed into the first or second hypoabelian group according as

$$
\left(\frac{2}{2 m+1}\right)^{n}=+1 \text { or }-1 \text {. }
$$

Next, $\xi_{0}^{2}+Q_{2 m}$ can be reduced to $\xi_{0}^{2}+f_{0}$, since the operation

$$
\xi_{0}^{\prime}=\xi_{0}+\xi_{1}+\xi_{2}, \quad \xi_{i}^{\prime}=\xi_{i}
$$

replaces $\xi_{0}^{2}+f_{c}$ by $\xi_{0}^{2}+f_{c+1}$. Now for $p=2, q / 2$ odd, the analysis in $\S 3$ failed to exhibit a quadratic invariant for the group on $y_{1}, \cdots, y_{q-2}$, but led to the invariant $\phi_{q-2}(y)+y_{q-1}^{2}$ for the group on $y_{1}, \cdots, y_{q-1}$. Hence the latter group can be transformed into one with the invariant

$$
y_{1} y_{2}+y_{3} y_{4}+\cdots+y_{q-3} y_{q-2}+y_{q-1}^{2} \text {. }
$$

* The results of $\$ \S 5,6$, are not employed in the subsequent sections. 
By an elementary discussion (the writer's Linear Groups, p. 200), the group is seen to be simply isomorphic with an abelian linear group on $q-2$ variables. An independent proof follows from $\S 6$.

Theorem. There exist representations of the symmetric group on $q$ letters as linear homogeneous groups as follows:

(i) for $q$ odd, a first or second hypoabelian group in the $G F\left[2^{n}\right]$ on $q-1$ variables, according as $(2 / q)^{n}=+1$ or -1 ;

(ii) for $q=4 l>4$, a first or second hypoabelian group in the $G F\left[2^{n}\right]$ on $q-2$ variables, according as $[2 /(q-1)]^{n}=+1$ or -1 ;

(iii) for $q=4 l+2>2$, an abelian linear group in any field $F_{2}$ having modulus 2 on $q-2$ variables.

By considering the monodromy group of the equation for the bisection of the periods of certain abelian functions, JoRDAN has shown (Traité des Substitutions, p. 364, § 498) that the symmetric group on $q$ letters is simply isomorphic with an abelian linear group modulo 2 on $2 k$ variables, $k$ being the greatest integer in $\frac{1}{2}(q-1)$. The exceptional character of the case $q=4$ was overlooked. The theorem obtained above shows that if $q \neq 4 l+2$ the abelian group may be taken to be a hypoabelian group.

Additional properties of our linear groups may be obtained by employing the further invariants* (5). Proceeding with $\omega_{3}$ and $\omega_{4}$ as we did with $\omega_{2}$ in $\S 3$, we obtain functions independent of $y_{q-1}$ if and only if $p=2$ and $p=3$, respectively. Thus for $p=2,3$, the group $L_{q-2, p}$ has the respective invariants

$$
\sum y_{j}^{2} y_{k}, \sum y_{j}^{2} y_{k} y_{l} \quad(j, k, l=1, \cdots, q-2 ; j, k, l \text { distinct; } k<l) .
$$

6. The fact that the symmetric group on $2 k$ letters $(2 k>4)$ is simply isomorphic with an abelian group modulo 2 on the variables $\xi_{i}, \eta_{i}(i=1, \ldots, k-1)$ may also be shown by the following correspondence of generators :

$$
\begin{aligned}
{[12] \sim L_{1}^{\prime},[23] \sim L_{1},[34] \sim V_{12},[45] \sim L_{2},[56] \sim V_{23}, \cdots, } \\
{[2 i, 2 i+1] \sim L_{i},[2 i+1,2 i+2] \sim V_{i i+1}, \cdots, } \\
{[2 k-2,2 k-1] \sim L_{k-1},[2 k-1,2 k] \sim L_{k-1}^{\prime}, }
\end{aligned}
$$

in which the $L$ 's and $V$ 's alternate. Here

$$
\begin{aligned}
& L_{i}: \xi_{i}^{\prime}=\xi_{i}+\eta_{i} ; \quad L_{i}^{\prime}: \eta_{i}^{\prime}=\eta_{i}+\xi_{i} ; \\
& V_{i j} \equiv V_{j i}: \eta_{i}^{\prime}=\eta_{i}+\xi_{i}+\xi_{j}, \eta_{j}^{\prime}=\eta_{j}+\xi_{i}+\xi_{j} .
\end{aligned}
$$

Since each transformation is of period 2 , and $V_{i j}$ is commutative with $L_{i}^{\prime}, L_{j}^{\prime}$, $V_{j k}$, while $L_{i}^{\prime} L_{i}$ and $L_{i} V_{i j}$ are of period 3 , the above transformations satisfy

* If we assume that the variables, as well as the coefficients, are integers taken modulo 2, then the group $L_{q-2,2}$ has, for $q=8 t+2$, the invariant 
Moore's set* of generational relations for the abstract form of the symmetric group. If we transform by $L_{1} L_{2} \ldots L_{k-1}$, we obtain the generators

$$
M_{1}, L_{1}, W_{12}, L_{2}, W_{23}, L_{3}, \cdots, L_{k-1}, M_{k-1},
$$

and hence have a direct generalization of the result for $k=6$ in Linear Groups,

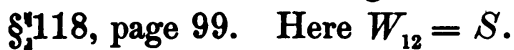

For $k=4$, the group generated by $L_{1}^{\prime}, L_{1}, \ldots$ has the (single) quadratic invariant $\dagger$

$$
\xi_{1} \eta_{1}+\xi_{2} \eta_{2}+\xi_{3} \eta_{3}+\xi_{1}+\xi_{2}+\xi_{3}+\eta_{1}+\eta_{3} \text {. }
$$

The abelian transformation $L_{2}^{\prime} L_{1}^{\prime} L_{3} Q_{1 \mathrm{~s}}$ replaces this by

$$
\xi_{1} \eta_{1}+\xi_{2} \eta_{2}+\xi_{3} \eta_{3} \text {. }
$$

Hence (in accord with $\S 5) S_{8 !}$ is representable as a first hypoabelian group. But the total first hypoabelian group modulo 2 on 6 variables is of order 8 !.

TheоRem. $\ddagger$ The symmetric group on 8 letters is simply isomorphic with the total first hypoabelian group moduln 2 on 6 variables.

7. Lemma. If the direct product of two groups $A$ and $B$ contains $a$ subgroup $G$ simply isomorphic with the symmetric group on $n$ letters, then $A$ or $B$ contains a subgroup of that type.

Let the operations of $G$ be $a_{1} b_{1}, a_{2} b_{2}, \ldots$. Then the distinct $a$ 's form a group $\alpha$, the distinct $b$ 's a group $\beta$. The largest group $\alpha^{\prime}$ common to $\alpha$ and $G$ is evidently invariant in each; the largest group $\beta$ common to $\beta$ and $G$ is invariant in each. As well known, the quotient-groups

$$
\frac{G}{\left\{\alpha^{\prime}, \beta^{\prime}\right\}}, \quad \frac{\alpha}{\alpha^{\prime}}, \quad \frac{\beta}{\beta^{\prime}}
$$

are simply isomorphic. By hypothesis, $G$ is simply isomorphic with $S_{\nu}, \nu=n !$. Hence the only invariant subgroups of $G_{\nu}$ are itself, the identity $I$, the group. $G_{i v}$ of the alternating type, and for $n=4$ a "four group" $G_{4}$. The lemma is obvious if either $\alpha^{\prime}$ or $\beta^{\prime}$ is $G$ itself, or if each is $I$, or if each is $G_{l v}$ (whence $n=2)$. Next let $\alpha^{\prime}=G_{i v}, \beta^{\prime}=I$. Then $G$ is composed of the operations of $\alpha^{\prime}=G_{1 v}$ and the products of $\frac{1}{2} \nu$ further $a^{\prime}$ s by an operation $b$ of period 2, so that $G$ is simply isomorphic with $\alpha$. Finally, if $n=4$, and $\alpha^{\prime}=G_{1}$, then $\beta^{\prime}$ cannot be of type $G_{4}$ or $G_{1 p}$, so that $\beta^{\prime}=I$; hence $\alpha$ is of order $4 !$ and is simply isomorphic with $G$.

* Proceedings of the London Mathematical Society, vol. 28 (1896), p. 357. For $k>2$ the isomorphism is simple in view of his theorem $C$.

$\dagger$ For $k=5$, this abelian group on 8 variables has no linear or quadratio invariant. The subgroup corresponding to the alternating group on $1, \cdots, 10$ has no quadratio invariant.

$\ddagger$ For a proof based on other principles, see the writer's note in the Bulletin of the American Mathematioal Sooiety, vol. 13, (1907), pp. 386-389. 
8. Theorem. For $q$ odd, the symmetric group on $q$ letters cannot be represented as a linear homogeneous group on $q-2$ variables with coefficients in a finite or infinite field $\boldsymbol{F}_{2}$ having modulus 2.

It follows from the theory of canonical forms that every transformation of period 2 of the general linear homogeneous group $H$ on $q-2$ variables in $F_{2}$ is conjugate within $H$ with one of the transformations

$$
\xi_{i}^{\prime}=\xi_{i}, \quad \xi_{r+i}^{\prime}=\xi_{r+i}+\xi_{i}, \quad \xi_{j}^{\prime}=\xi_{j} \quad(i=1, \cdots, r ; j=2 r+1, \cdots, q-2),
$$

where $r$ is an integer such that $2 r \leqq q-2$, whence $r \leqq \frac{1}{2}(q-3)$. The matrix of (15) and the matrix of the general transformation of $H$ commutative with (15) may be given the compact notations

$$
\begin{array}{llllll}
I & O & O & A & O & O \\
I & I & O, & C & A & D, \\
O & O & I & E & O & B
\end{array}
$$

in which each $I$ is a unit or identity matrix, each $O$ a matrix all of whose elements are zero, while $A$ and $B$ are square matrices of orders $r$ and $q-2-2 r$, respectively.

Suppose now that $H$ has a subgroup $G_{q \text { ! }}$ of symmetric type. We may assume that the latter contains a transformation (15) corresponding to the transposition $\left[l_{1} l_{2}\right]$, and that the group $K$ of the transformations $\left(1_{2}\right)$ contains a subgroup $G_{\omega}, \omega=(q-2)$ !, simply isomorphic with the symmetric group on $l_{3}, \ldots, l_{q}$. The group $K$ has the invariant subgroup $J$,

$$
\begin{aligned}
& \begin{array}{lllllll}
I & O & O & & A & O & O
\end{array} \\
& J: \quad C \quad I \quad D, \quad Q: O A \quad O \text {, } \\
& \begin{array}{llllll}
E & O & I & O & O & B
\end{array}
\end{aligned}
$$

the quotient group $K / J$ being simply isomorphic with $Q$. The largest group common to $J$ and $G_{\omega}$ is invariant in the latter. Now every transformation of $J$ is of order a power of 2 . But, for $n \neq 4, S_{n !}$ contains no invariant subgroup of order a power of 2 . Since $q$ is odd, it follows that $G_{\omega}$ is simply isomorphic with a subgroup of $Q$, and hence $(\S 7)$ with a subgroup of one of the linear groups $A$ and $B$ on $r$ and $q-2-2 r$ variables, respectively. Since $1 \leqq r \leqq \frac{1}{2}(q-3)$, we bave $q-2-2 r \leqq q-4$, and, if $q \geqq 5, r \leqq q-4$. Hence the theorem follows by induction from $q-2$ to $q$, it being obviously true for $q=3$, since a unary homogeneous group is commutative.

For $q$ even, we apply the preceding theorem to the subgroup leaving $l_{q}$ fixed and obtain the

Corollary. For $q$ even, $S_{q 1}$ cannot be represented as a linear homogeneous group in $F_{2}$ on $q-3$ variables. 
The preceding theorem and corollary form a sequel to the results of $\S \S 2,4$ for the case when the modulus is 2 .

9. Henceforth we discuss the representations of $S_{q !}$ as an $m$-ary linear homogeneous group in a field $F$ not having modulus 2 . Within the general linear homogeneous group $G L H(m, F)$, any transformation of period 2 is conjugate with one of the form $\Pi C_{i}$, where $C_{i}$ alters only $\xi_{i}$, whose sign it changes. We thus assume that the transposition [12] corresponds to $C_{1} C_{2} \ldots C_{r}$. Then each transposition corresponds to a transformation $\left(\alpha_{i j}\right)$ with the characteristic determinant

$$
|\alpha-\rho I|=(-1-\rho)^{r}(1-\rho)^{m-r} \text {. }
$$

To [34] corresponds a transformation $S$ commutative with $C_{1} \ldots C_{r}$. Transforming $S=S_{1, \ldots, r} S_{r+1, \ldots, m}^{\prime}$ by a transformation of type $T_{1, \ldots, r} T_{r+1, \ldots, m}^{\prime}$, we obtain a product of $r$ of the $C_{i}$. Hence*

$$
[12] \sim C_{1} \ldots C_{r}, \quad[34] \sim C_{1} \cdots C_{1} C_{r+1} \ldots C_{2 r-s} \quad(0 \leqq s<r) .
$$

Since $[13][24]$ is commutative with $[12][34]$, we get

$$
[13][24] \sim R S_{1, \ldots, s, 2 r-8+1, \ldots, m}, \quad R=\left(\begin{array}{cc}
\alpha & \beta \\
\gamma & \delta
\end{array}\right),
$$

where $\alpha, \cdots, \delta$ are square matrices of order $r-s, \alpha$ affecting the variables $\xi_{s+1}, \cdots, \xi_{r}$, and $\delta$ the variables $\xi_{r+1}, \ldots, \xi_{2 r-s^{\circ}}$ We may obtain [14] [23] by transforming [13] [24] by [12] or as the product [13][24] $[12][34]$. Identifying the two results, we find that $\alpha=\delta=0$, and that $S$ is commutative with $C_{1} \ldots C_{\text {. }}$. Also $\beta \gamma=\gamma \beta=I$, since $R^{2}=I$. Hence

$$
[13][24] \sim\left(\begin{array}{cc}
O & \beta \\
\beta^{-1} & O
\end{array}\right) \cdot S_{1, \ldots, s} S_{2 r-8+1, \ldots, m}^{\prime} .
$$

We may take $\beta=I, S=C_{1} \cdots C_{\sigma}, S^{\prime}=C_{2 r-0+1} \cdots$, by transforming by

$$
(\beta)_{r+1, \ldots, 2 r-8} T_{1, \ldots, 8} T_{2 r-8+1, \ldots, m}^{\prime},
$$

where $T$ and $T^{\prime}$ are suitably chosen. Then (18) are unaltered, and

$$
[13][24] \sim C_{1} \ldots C_{\sigma} \prod_{i=1}^{r-8}\left(\xi_{\sigma+i} \xi_{r+i}\right) \prod_{i=1}^{r-s-\sigma} C_{2 r-o+i} \quad(0 \leqq 0 \leqq 8, \sigma \leqq r-8) .
$$

The number of factors in the final product must be $r-s-\sigma$ since the number of roots -1 of the characteristic equation is $2 r-2 s$, [13] [24] being conjugate with [12] [34]. By (18) and (19), we have

$$
[14][23] \sim C_{1} \ldots C_{\sigma} \prod_{i=1}^{r-8}\left(\xi_{s+i} \xi_{r+i}\right) \prod_{i=8+1}^{3 r-2 s-\sigma} C_{i}
$$

* The terms $C_{1} \cdots C_{8}$ are to be suppressed in the case $s=0$. Similarly below. For the caco $q=3$, here excluded, we may proceed as in 816 , with $k=0$. 
In applying (18) and (19) simultaneously, it is convenient to have the variables separated into the sets $\xi_{i_{1}}, \ldots, \xi_{i_{6}}$, where

$$
\begin{array}{r}
i_{1}=1, \ldots, \sigma ; i_{2}=\sigma+1, \ldots, s ; i_{3}=s+1, \ldots, r ; \\
i_{4}=r+1, \ldots, 2 r-s ; i_{5}=2 r-s+1, \ldots, 3 r-2 s-\sigma ; \\
i_{6}=3 r-2 s-\sigma+1, \ldots, m ;
\end{array}
$$

the set $\xi_{i_{1}}$ not occurring if $\sigma=0$, etc. Then (20) may be written

$$
[14][23] \sim(-I)_{i_{1}}(I)_{i_{2}}\left(\begin{array}{rr}
O & -I \\
-I & O
\end{array}\right)_{i_{3}, i_{4}}(-I)_{i_{5}}(I)_{i_{6}} .
$$

Let [23] $K=\left(\kappa_{a b}\right), a, b=1, \cdots, 6$. Since $K$ is commutative with (20'),

$$
K=\left(\begin{array}{cccccc}
\kappa_{11} & O & \kappa_{12} & \kappa_{13} & \kappa_{15} & 0 \\
O & \kappa_{22} & \kappa_{23} & -\kappa_{23} & O & \kappa_{26} \\
\kappa_{31} & \kappa_{32} & \kappa_{33} & \kappa_{34} & \kappa_{35} & \kappa_{36} \\
\kappa_{31} & -\kappa_{32} & \kappa_{34} & \kappa_{33} & \kappa_{35} & -\kappa_{36} \\
\kappa_{51} & O & \kappa_{53} & \kappa_{53} & \kappa_{55} & O \\
O & \kappa_{62} & \kappa_{63} & -\kappa_{63} & O & \kappa_{66}
\end{array}\right] .
$$

Now [14] may be obtained by transforming [23] by [13][24] and also by multiplying [23] by [14][23]. Equating the results for the corresponding transformations, we get

$$
\kappa_{11}=\kappa_{15}=\kappa_{23}=\kappa_{32}=\kappa_{36}=\kappa_{51}=\kappa_{55}=\kappa_{63}=0, \quad \kappa_{34}=-\kappa_{33} .
$$

Hence, setting $\kappa=\kappa_{33}$, we have

$$
K=U V, \quad U=\left(\begin{array}{cccc}
O & \kappa_{13} & \kappa_{13} & O \\
\kappa_{31} & \kappa & -\kappa & \kappa_{35} \\
\kappa_{31}-\kappa & \kappa & \kappa_{35} \\
O & \kappa_{63} & \kappa_{53} & O
\end{array}\right)_{i_{1}, i_{3}, i_{4}, i_{5}}, \quad V=\left(\begin{array}{ll}
\kappa_{22} & \kappa_{26} \\
\kappa_{62} & \kappa_{66}
\end{array}\right)_{i_{2}, 4}
$$

We shall employ the symbols

$$
\left.\mu \equiv \begin{array}{c}
\kappa_{13} \\
\kappa_{53}
\end{array}\right\}, \quad \nu \equiv \overbrace{\kappa_{31} \kappa_{35}}
$$

to denote the square matrices of order $r-s$ defined as follows: The first $\sigma$ rows of $\mu$ are composed of the elements of $\kappa_{13}$, the remaining $r-s-\sigma$ rows are composed of the elements of $\kappa_{53}$; while $\nu$ is formed by annexing columns. By 
combining the sets of variables $\xi_{i_{1}}$ and $\xi_{i_{6}}$ into one set $\xi_{i_{1,5}}$, we have

Hence $U^{2}=I$ if and only if

$$
U=\left(\begin{array}{rrr}
\kappa & -\kappa & \nu \\
-\kappa & \kappa & \nu \\
\mu & \mu & O
\end{array}\right)_{i_{8,}, i_{4,} i_{1,5}}
$$

$$
\mu \nu=\frac{1}{2} I, \quad \kappa^{2}=11 .
$$

Now [12] [23] is of period 3. Hence must

$$
\left(C_{\sigma+1} \ldots C, V\right)^{3}=I
$$

and $(C U)^{3}=I$, where $C=C_{1} \ldots C_{\sigma} C_{s+1} \ldots C_{r}$. For $U$ given by (22), the conditions for $(C U)^{2}=U C$ are seen to be

$$
2 \kappa_{13} \kappa=-\kappa_{13}, 2 \kappa_{53} \kappa=\kappa_{53}, 2 \kappa \kappa_{31}=-\kappa_{31}, 2 \kappa \kappa_{35}=\kappa_{35}, \kappa_{35} \kappa_{53}-\kappa_{31} \kappa_{13}=\kappa .
$$

The first two of these conditions give

$$
\mu \kappa=\delta \mu,
$$

where $\delta$ is a square matrix all of whose non-diagonal elements are zero, while in the main diagonal the first $\sigma$ elements are $-\frac{1}{2}$, and the remaining $r-s-\sigma$ elements are $+\frac{1}{2}$.

We next normalize the linear group by a transformation of variables. We multiply all matrices on the left by $M$ and on the right by $M^{-1}$, where $M=(\mu)_{i_{3}}(\mu)_{i_{4}}$. Evidently (18)-(20) are unaltered. Then (24) becomes a matrix in which $\kappa, \mu, \nu$ are replaced by $\mu \kappa \mu^{-1}, I, \mu \nu$, respectively. Hence by $\left(25_{1}\right)$ and $(28)$, the new $U$ is

$$
U=\left(\begin{array}{rrr}
\delta & -\delta & \frac{1}{2} I \\
-\delta & \delta & \frac{1}{2} I \\
I & I & O
\end{array}\right)_{i_{3}, \varepsilon_{4}, i_{1}, 5}
$$

The remaining conditions $\left(25_{2}\right)$ and $(27)$ are seen to be satisfied. The characteristic equation of (29) is seen to have -1 as a root of multiplicity $r-s+\sigma$. Hence by (17) that of $V$ has -1 as root of multiplicity $s-\sigma$.

TheOREM. In every representation of the symmetric group $S_{41}$ as a linear homogeneous group in a field not having modulus 2, we may, after a suitable transformation of variables, take the correspondence of generators to be (18)-(20) and [23] UV, where $U$ is given by (29) and $V$ is a transformation $V_{i_{2,6}}$ subject to condition (26) and having -1 as a root of multiplicity s $-\sigma$ of its characteristic equation.

10. A transformation is commutative with (18), (19), and $U V$ if and only 
if it has the form

$$
T_{i_{3}} T_{i_{4}} T_{i_{1}, 5} Q_{i_{2}} R_{i_{6}}, \quad T=\left(\begin{array}{cc}
A & O \\
O & . B
\end{array}\right),
$$

where $A$ and $B$ are square matrices of orders $\sigma$ and $r-s-\sigma$, respectively.

If $q \geqq 6$, let [56 ] $S$. By transforming the linear group by a transformation of type (30), we may express $S$ as a product of $C$ 's, without disturbing the earlier correspondences. Hence we may set

$$
\text { [56] } \prod_{i=1}^{\sigma_{1}} C_{s+i} C_{r+i} C_{i} \cdot \prod_{i=1}^{\sigma_{8}} C_{s+\sigma+i} C_{r+\sigma+i} C_{2 r-\sigma+i} \cdot \prod_{i=1}^{\sigma_{2}} C_{\sigma+i} \cdot \prod_{i=1}^{\sigma_{4}} C_{3 r-2 s+\sigma+i}
$$

where

$$
\begin{aligned}
& 0 \leqq \sigma_{2} \leqq r-8-\sigma, \quad 0 \leqq \sigma_{3} \leqq 8-\sigma, \quad 0 \leqq \sigma_{4}, \\
& 3\left(\sigma_{1}+\sigma_{2}\right)+\sigma_{3}+\sigma_{4}=r \text {. }
\end{aligned}
$$

Now $\left(18_{1}\right)$ and (31) have $2 \sigma_{1}+\sigma_{2}+\sigma_{3}$ factors $C$ in common, while the products $(18)$ have $s$ factors in common. Hence

$$
2 \sigma_{1}+\sigma_{2}+\sigma_{3}=\text { s. }
$$

11. The main object of the paper is to determine the minimum number of variables upon which the symmetric group $S_{q !}$ can be represented. For this purpose it will be seen $(\$ \S 19,20)$ to be sufficient to know the representations in the case $r=1$, whence $s=\theta$. We shall assume merely that $s=0$, as the specialization of $r$ does not materially simplify the discussion.

For $s=0$, we have $\sigma=0$. Then $V=I$ since $V^{2}=I$ and since all the roots of the characteristic equation are +1 . Hence

$$
[12] \sim C_{1} \ldots C_{r}, \quad[34] \sim C_{r+1} \ldots C_{2 r},
$$$$
[23] \sim U_{1, \ldots, 3 r},
$$

the matrix of $U$ being given by (29) for $\delta=\frac{1}{2} I$. Hence

$$
\begin{gathered}
U_{1, \ldots, 3 r}: \xi_{i}^{\prime}=\xi_{i}+\omega_{i}, \xi_{r+i}^{\prime}=\xi_{r+i}+\omega_{i}, \xi_{2 r+i}^{\prime}=\xi_{2 r+i}-2 \omega_{i} \quad(i=1, \cdots, r), \\
\omega_{i}=-\frac{1}{2} \xi_{i}-\frac{1}{2} \xi_{r+i}+\frac{1}{2} \xi_{2 r+i}, \quad \omega_{i}^{\prime}=-\omega_{i} \text { under } U .
\end{gathered}
$$

In $\S 10$ we now have $\sigma_{1}=\sigma_{2}=\sigma_{3}=0, \sigma_{4}=r$. Hence if $q \geqq 6$,

$$
\text { [56] } C_{3 r+1} \ldots C_{4} \text {. }
$$

Since [36] [45] is commutative with [12] and [34][56], and since its transform by [56] and its product by [34][56] both equal [35][46], we have

$$
[36][45] \sim T=A_{1, \ldots, r} B_{r+1, \ldots, 2 r, 3 r+1, \ldots, 4 r} C, \quad B=\left(\begin{array}{ll}
O & \beta \\
\beta^{-1} & 0
\end{array}\right),
$$

where $\beta$ is a square matrix of order $r$, and

$$
C=\left(\gamma_{i j}\right), \quad C^{2}=I \quad(i, j=2 r+1, \cdots, 3 r, 4 r+1, \cdots, m) .
$$


Transforming the group by $(\beta)_{3 r+1, \ldots, 4 r}$, we may set $\beta=I$. Then the characteristic determinant $B_{\rho}$ of $B$ equals $\left(\rho^{2}-1\right)^{r}$. If $A_{\rho}=0$ has $t$ roots -1 , then $C_{\rho}=0$ has $r-t$ roots -1 , since [36] [45] is conjugate with [12] [34]. Then [12] [36] [45] has $r-t+r+r-t$ roots -1 , but is conjugate with [12] [34] [56] with $3 r$ roots -1 ; hence $t=0, A=I$. Thus

$$
[36][45] \sim T=\prod_{i=1}^{r}\left(\xi_{r+i} \xi_{3 r+i}\right) \cdot C \quad\left(c_{\rho} \text { with } r \text { roots }-1\right) .
$$

Let $[45] \sim L$. We shall determine $L$ by a device which brings to light its essential properties. The product

$$
[36][45] \cdot[23] \cdot[34] \cdot[56]
$$

transforms [23] into [45]. Let $K=C_{r+1} \ldots C_{2 r} C_{3 r+1} \ldots C_{4 r}$. Then the product $P=T U K$ transforms $U$ into $L$. Hence $P^{-1}=K U T$ replaces the invariants of $U$ by invariants of $L$, and replaces $\omega_{i}$, given by (37), by a function which $L$ must multiply by -1 , namely,

$$
\tau_{i}=-1 \xi_{i}-1 \xi_{r+i}-1 \xi_{2 r+i}+\frac{1}{2} \xi_{3 r+i}+\frac{1}{2} \sum_{j=1}^{r} \gamma_{2 r+i, 2 r+j}\left(\xi_{j}-\xi_{r+j}\right)+\frac{1}{2} \sum_{j=4 r+1}^{m} \gamma_{2 r+i, j} \xi_{j} .
$$

Applying $P^{-1}$ to the invariants $\xi_{r+i}+\frac{1}{2} \omega_{i}$ and $\xi_{3 r+i}$ of $U$, we get

$$
-\xi_{3 r+i}+\frac{1}{2} \tau_{i}, \omega_{i} \quad(i=1, \cdots, r) .
$$

Since $C$ replaces $\sum \gamma_{i j} \xi_{j}$ by $\xi_{i}$ by (39), $P^{-1}$ replaces the invariants

$$
\sum_{j=1}^{r} \gamma_{i, 2 r+j}\left(\xi_{2 r+j}-\omega_{j}\right)+\sum_{j=4 r+1}^{m} \gamma_{i j} \xi_{j} \quad(i=2 r+1, \cdots, 3 r, 4 r+1, \cdots, m)
$$

of $L^{r}$ by the following invariants of $L$ :

$$
\xi_{i}-\xi_{r+i}-\sum_{j=1}^{r} \gamma_{2 r+i, 2 r+j} \tau_{j}(i=1, \cdots, r), \quad \xi_{i}-\sum_{j=1}^{r} \gamma_{i, 2 r+j} \tau_{j}(i=4 r+1, \cdots, m) .
$$

Since [45] is commutative with [12], $L$ must be commutative with $C_{1} \ldots C_{r}$. Hence $L$ replaces $\xi_{1}, \ldots, \xi_{r}$ by functions of the same, while the function by which $L$ replaces $\xi_{t}(t>r)$ is independent of $\xi_{1}, \ldots, \xi_{r}$. Hence by the invariance of $\omega_{1}, \ldots, \omega_{r}, L$ leaves fixed $\xi_{1}, \ldots, \xi_{r}$. But $L$ must multiply each $\tau_{i}$ by -1 ; hence the $\tau_{i}$ are independent of $\xi_{1}, \cdots, \xi_{r}$, so that

$$
\gamma_{2 r+i, 2 r+i}=\frac{1}{2}, \quad \gamma_{2 r+i, 2 r+j}=0 \quad(i, j=1, \cdots, r ; i \neq j) .
$$

Suitable linear combinations of the above invariants of $L$ give

$$
\xi_{i}, \xi_{r+i}+\frac{1}{2} \tau_{i}, \xi_{2 r+i}-\xi_{r+i}, \xi_{3 r+i}-\frac{1}{2} \tau_{i} \quad(i=1, \cdots, r) .
$$


In view of these and $\left(41_{2}\right)$, the explicit form of $L$ is

$$
\begin{aligned}
& \delta \xi_{r+i}=\tau_{i}, \quad \delta \xi_{2 r+i}=\tau_{i}, \quad \delta \xi_{3 r+i}=-\tau_{i}(i=1, \cdots, r), \quad \delta \xi_{i}=-2 \sum_{j=1}^{r} \gamma_{i, 2 r+j} \tau_{j} \\
&(i=4 r+1, \cdots, m),
\end{aligned}
$$

where $\delta \xi \equiv \xi^{\prime}-\xi$. Now $L$ actually multiplies each $\tau_{i}$ by -1 if and only if*

$$
\sum_{j=4 r+1}^{m} \gamma_{2 r+i, j} \gamma_{j, 2 r+k}=\frac{3}{4} \delta_{i k} \quad(i, k=1, \cdots, r),
$$

where, in KroneCKER's notation, $\delta_{i i}=1, \delta_{i k}=0(i \neq k)$.

Since $L$ was shown to leave each $\omega_{i}$ invariant, and since $U$ evidently leaves each $\tau_{i}$ invariant, $L$ and $U$ are commutative. Since $L C_{r+1} \ldots C_{2 r}$ and $L C_{3 r+1} \ldots C_{4 r}$ replace $\tau_{i}$ by $\xi_{r+i}$ and $-\xi_{3 r+i}$, respectively, they are easily seen to have period 3. Hence the correspondence given by (35), (38), and [45] $L$, define a simple isomorphism between $S_{6 !}$ and an $m$-ary linear group.

12. We may now readily treat the case when $q$ is general :

$$
q=4 \kappa+\kappa^{\prime} \quad\left(0 \leqq \kappa^{\prime}<4\right) .
$$

By the proof for [56], any transposition $[i j], i>4, j>4$, corresponds to a transformation on the variables $\xi_{k}(k>3 r)$. Hence we may set

$$
\begin{gathered}
{[56] \sim \Pi C_{3 r+i}, \quad[67] \sim U_{3 r+1, \ldots, 6 r}, \quad[78] \sim \Pi C_{4 r+i} ;} \\
{[9,10] \sim \Pi C_{6 r+i}, \quad[10,11] \sim U_{6 r+1, \ldots, 9 r}, \quad[11,12] \sim \Pi C_{i r+i} ; \ldots} \\
{[4 \kappa-3,4 \kappa-2] \sim \Pi C_{(3 \kappa-3)+i}, \quad[4 \kappa-2,4 \kappa-1] \sim U_{(3 \kappa-3) r+1, \ldots, 3 \kappa r},} \\
{[4 \kappa-1,4 \kappa] \sim \Pi C_{(3 \kappa-2) r+i},}
\end{gathered}
$$

where for each product $i=1, \ldots, r$. Each of the $\kappa$ "triples"'(35) and (47) defines a simple isomorphism between the symmetric group on four letters and a linear group on $3 r$ variables. It remains to insert the connecting " links" [45] $\sim L_{1},[89] \sim L_{2}, \ldots$, and when $\kappa^{\prime}>0$ in (46) to extend the series beyond these $\kappa$ triples ( $\$ 14-16)$.

In $\S 11$ the only normalization was the transformation by $(\beta)_{3 r+1, \ldots, 4 r}$. We here accomplish this normalization by transforming the linear group by

$$
(\beta)_{3 r+1, \ldots, 4 r}(\beta)_{4 r+1, \ldots, 5 r}(\beta)_{5 r+1, \ldots, 6 r},
$$

and hence preserve the correspondences (35) and (47). Then $L_{1}$ is given by (43), subject to (44) and (45). Now $L_{1}$ must be commutative with all the transformations (47) except the first. Thus $L_{1}$ does not affect the variables

- These conditions aleo follow from (39) and (42). 
$\xi_{i}(i=6 r+1, \ldots, 3 \kappa r)$, in view of the triples other than the first, nor $\xi_{i}(i=4 r+1, \cdots, 5 r)$, in view of [78]. Finally, $L_{1}$ must be commutative with $U=U_{3 r+1, \ldots, o r}$. The necessary and sufficient conditions are that $U$ leave unaltered each $\tau_{i}$ and that $L_{1}$ leave unaltered the functions

$$
-\frac{1}{2} \xi_{3 r+i}-\frac{1}{2} \xi_{4 r+i}+\frac{1}{2} \xi_{b r+i} \quad(i=1, \cdots, r),
$$

which $U$ multiplies by -1 . Hence

$$
\gamma_{2 r+i, b r+j}=\frac{1}{2} \delta_{i j}, \quad \gamma_{b r+i, 2 r+j}=\frac{1}{2} \delta_{i j} \quad(i, j=1, \cdots, r) .
$$

Then relations (45) become

$$
\sum_{j=8 k r+1}^{m} \gamma_{2 r+i, j} \gamma_{j, 2 r+k}=\frac{1}{2} \delta_{i k} \quad(i, k=1, \cdots, r) .
$$

In the final terms of (43) and (44) we may restrict $i$ and $j$ to the values $3 \kappa r+1, \cdots, m$, provided we insert the term $1 \xi_{5 r+i}$ in $(44)$, and $8 \xi_{5 r+l}=-\tau_{i}$ in (43).

We next exhibit $L_{1}$ in a notation better suited for the further discussion. We set

$$
m=3 \kappa r+\mu, \quad \tau_{i}=\tau_{i 1}, \quad \gamma_{3 \kappa r+i, 2 r+j}=\frac{1}{2} \beta_{i 11}, \quad \gamma_{2 r+j, 3 \times r+i}=\frac{1}{2} \alpha_{j 1 i},
$$

for $i=1, \ldots, \mu ; j=1, \ldots, r$. Then

$$
\begin{array}{rr}
L_{1}: \delta \xi_{r+i}=\delta \xi_{2 r+i}=\tau_{i 1}, \quad \delta \xi_{3 r+i}=\delta \xi_{5 r+i}=-\tau_{i 1} & (i=1, \cdots, r), \\
\delta \xi_{3 \times r+i}=-\sum_{j=1}^{r} \beta_{i j 1} \tau_{j 1} & (i=1, \cdots, \mu), \\
\tau_{i 1}=-\frac{1}{2} \xi_{r+i}-\frac{1}{4} \xi_{2 r+i}+\frac{1}{2} \xi_{3 r+i}+\frac{1}{4} \xi_{5 r+i}+1 \sum_{j=1}^{\mu} \alpha_{i j j} \xi_{3 \times r+j} & (i=1, \cdots, r), \\
\sum_{j=1}^{\mu} \alpha_{i j j} \beta_{j k 1}=2 \delta_{i k} & (i, k=1, \cdots, r) .
\end{array}
$$

The link $[4 t, 4 t+1] \sim L_{t}$ may be obtained from $L_{1}$ by adding $3(t-1) r$ to the subscripts $\leqq 3 \kappa r$ of the $\xi$ 's. Hence, for $t=1, \ldots, \kappa-1$,

$$
\begin{aligned}
& L_{i}: \delta \xi_{(3 t-2) r+i}=\delta \xi_{(3 t-1) r+i}=\tau_{i t}, \quad \delta \xi_{3 t+i}=\delta \xi_{(3 t+2) r+i}=-\tau_{i t} \quad(i=1, \cdots, r), \\
& \delta \xi_{3 k+j}=-\sum_{k=1}^{r} \beta_{j k t} \tau_{k t} \quad(j=1, \cdots, \mu), \\
& \tau_{i t}=-\frac{1}{2} \xi_{(3 t-2) r+i}-1 \frac{1}{4(3 t-1) r+i}+\frac{1}{2} \xi_{3 c r+i}+1 \xi_{(3 t+2) r+i}+1 \sum_{j=1}^{\mu} \alpha_{i j} \xi_{3 k r+j}, \\
& \sum_{j=1}^{\mu} \alpha_{i k j} \beta_{j k t}=2 \delta_{i k} \\
& (i, k=1, \cdots, r) \text {. }
\end{aligned}
$$

Now $L_{t}$ and $L_{t}$ are commutative if and only if each leaves fixed the $\tau_{i}$ functions belonging to the other. According as $s=t+1$ or $s>t+1$, the condi- 
tions are, respectively,

$$
\begin{aligned}
\sum_{j=1}^{\mu} \alpha_{i t+i j} \beta_{j k t} & =\delta_{i k}, & \sum_{j=1}^{\mu} \alpha_{i j} \beta_{j k t+1} & =\delta_{i k}, \\
\sum_{j=1}^{\mu} \alpha_{i j s} \beta_{j k t} & =0, & \sum_{j=1}^{\mu} \alpha_{i j} \beta_{j k s} & =0
\end{aligned}
$$

holding for $s, t=1, \ldots, \kappa-1 ; i, k=1, \cdots, r$.

13. The product of the two matrices

$$
A_{i}=\left(\alpha_{i t j}\right), \quad B_{k}=\left(\beta_{j k t}\right) \quad(t=1, \cdots, \kappa-1 ; j=1, \cdots, \mu),
$$

the first having $\kappa-1$ rows and $\mu$ columns and the second having $\mu$ rows and $\kappa-1$ columns, is a square matrix of order $\kappa-1$ :

$$
P_{i k}=\left(\pi_{t l}^{i k}\right), \quad \pi_{t l}^{i k} \equiv \sum_{j=1}^{\mu} \alpha_{i t j} \beta_{j k l} \quad(t, l=1, \cdots, k-1) .
$$

In view of (52)-(54), we have

$$
\pi_{t t}^{i k}=2 \delta_{i k}, \quad \pi_{t+1 t}^{i k}=\pi_{t t+1}^{i k}=\delta_{i k}, \quad \pi_{s t}^{i k}=\pi_{t o}^{i k}=0 \quad(8 \geqq t+2) .
$$

Hence if $i \neq k, P_{i k}$ is a matrix $O$ all of whose elements are zero. Also each $\boldsymbol{P}_{i \mathrm{i}}$ is the matrix

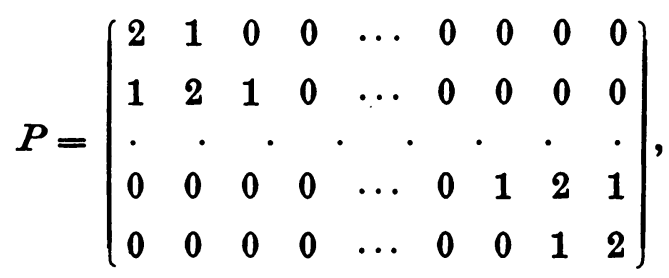

the elements in the main diagonal being 2 , those in the adjacent parallels being 1 , and all the remaining elements being 0 . If we set $|P|=\Delta_{k-1}$ and expand according to the first row, we have

$$
\Delta_{k-1}=2 \Delta_{k-2}-\Delta_{k-3},
$$

whence, by induction, $\Delta_{\kappa-1}=\kappa$. As in (23), we set

$$
\left.A=\begin{array}{c}
A_{1} \\
\dot{A}_{r}
\end{array}\right\}, \quad B=\overbrace{B_{1} \cdots B_{r}} .
$$

Since $A_{i} B_{k}=P_{i k}$, we have

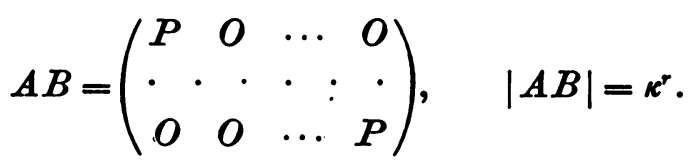

First, let $\mu<(\kappa-1) r$. We convert matrices $A$ and $B$ into square matrices. 
of order $(\kappa-1) r$ by adding columns of zero elements to the former and rows of zero elements to the latter. In view of their determinants, we have $0.0=\kappa^{r}$. Hence the field $F$ must have a modulus which divides $\boldsymbol{\kappa}$.

Next, let $\mu<(\kappa-2) r$. Drop the last row of each $A_{i}$ and the last column of each $B_{i}$. Let $A$ and $B$ then become $A^{*}$ and $B^{*}$. Hence

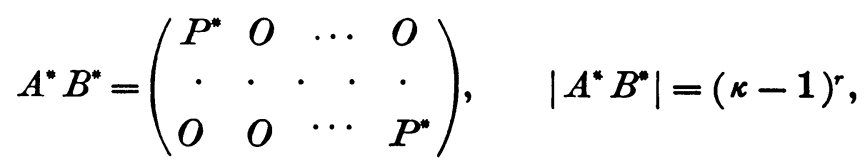

where $P^{*}$ is obtained from $P$ by deleting the last row and last column. Thus $\left|P^{*}\right|=\Delta_{\kappa-2}=\kappa-1$. We convert $A^{*}$ and $B^{*}$ into square matrices of order $(\kappa-2) r$ by adding columns of zero elements to the former and rows of zero elements to the latter. Hence $0.0=(\kappa-1)^{r}$, in contradiction to the preceding case.

Theorem. For $q \geqq 4 \kappa$ we have $\mu \geqq(\kappa-1) r$ or $\mu \geqq(\kappa-2) r$, according as the field does not have or has a modulus which divides $\kappa$.

By $§ \S 2-4$ the symmetric group on $4 \kappa$ letters can always be represented as a linear group $G$ on $4 \kappa-1$ variables; and, if the modulus divides $\kappa$, as a linear group $G^{\prime}$ on $4 \kappa-2$ variables. By notation, $m \equiv 3 \kappa r+\mu$. If $F^{\prime}$ does not have a modulus dividing $\kappa$, the direct product of $r$ groups $G$ gives a representation for which the number of variables is $(4 \kappa-1) r=m$, with $\mu=(\kappa-1) r$. If $F$ has a modulus dividing $\kappa$, the direct product of $l$ groups $G$ and $r-l$ groups $G^{\prime}$ gives a representation for which the number of variables is

$$
(4 \kappa-1) l+(4 \kappa-2)(r-l)=m, \quad \text { with } \quad \mu=(\kappa-2) r+l .
$$

Thus $\mu$ may have any of the values $(\kappa-2) r,(\kappa-2) r+1, \ldots$ In particular the limits which the theorem assigns to $\mu$ cannot be lowered.

The problem of the normalization of the groups is considered in $\S \S 22-24$.

14. Let next $q \geqq 4 \kappa+1$, and set $[4 \kappa-1,4 \kappa+1] \sim S$. Then $S$ must be commutative with the first $\kappa-1$ triples (35) and (47), and with $\Pi C_{(3 \kappa-3) r+i}$. The variables in the latter are not affected by $S$ in view of the hypothesis of $\S 11$. Hence

$$
S: \quad \xi_{(3 \kappa-2) r+i}^{\prime}=\sum_{j=1}^{\nu} \sigma_{i j} \xi_{(3 \kappa-2) r+j}
$$$$
(i=1, \cdots, v),
$$

where $\nu=m-(3 \kappa-2) r$. Now

Hence

$$
[4 \kappa-1,4 \kappa] \sim P=\prod_{i=1}^{r} C_{(3 \kappa-2) r+i}
$$

$$
[4 \kappa, 4 \kappa+1] \sim T=S P^{\prime} S: \delta \xi_{(3 \kappa-2) r+i}=-2 \sum_{i=1}^{r} \sigma_{i j} \tau_{j} \quad(i=1, \cdots, \nu),
$$


in which $\tau_{i}$ denotes the sum (60) for $i \leqq r$. Next, $T$ must be commutative with

$$
\begin{aligned}
U_{(3 \kappa-3) r+1, \ldots, 3 k}: & \delta \xi_{(3 \kappa-3) r+i}=\delta \xi_{(3 \kappa-2) r+i}=\lambda_{i}, \quad \delta \xi_{(3 \kappa-1) r+i}=-2 \lambda_{i}, \\
\lambda_{i} & =-\frac{1}{2} \xi_{(3 \kappa-3) r+i}-\frac{1}{2} \xi_{(3 \kappa-2) r+i}+\frac{1}{2} \xi_{(3 \kappa-1) r+i} \quad(i=1, \ldots, r),
\end{aligned}
$$

in accord with (36), (37). But $U T$ and $T U$ replace $\xi_{(3 k-3) r+i}$ by equal functions if and only if $T$ leaves $\lambda_{i}$ unaltered, the conditions for which are

$$
\sigma_{i j}=\sigma_{r+i j} \quad(i, j=1, \cdots, r) .
$$

Again, $U T$ and $T U$ replace $\xi_{(3 k-2) r+i}$ by equal functions if and only if $U$ leaves unaltered the sum in (61). For $i=1, \cdots, \nu, r$ of these sums are linearly independent since the $\sigma_{i j}$ form the first $r$ columns of the matrix of $S$. Hence $U$ must leave unaltered each $\tau_{j}$, so that

$$
\sigma_{j i}=2 \sigma_{j r+i} \quad(i, j=1, \cdots, r) .
$$

Next, $P$ must transform $T$ into $S$. The conditions are

$$
\delta_{i k} \pm 2 \sum_{j=1}^{r} \sigma_{i j} \sigma_{j k}=\sigma_{i k} \quad(i, k=1, \cdots, \nu) .
$$

the sign being minus if and only if $i$ and $k$ are both $\leqq r$ or both $>r$. Let first $k \leqq r, i=r+\epsilon, \epsilon \leqq r$; then, by (62), (64) becomes

$$
2 \sum_{j=1}^{r} \sigma_{e j} \sigma_{j k}=\sigma_{\epsilon k}
$$

Adding this to (64) for $i=\epsilon$, we get $\frac{1}{2} \delta_{e k}=\sigma_{\epsilon k}$. Hence by (62), (63),

$$
\sigma_{i j}=\sigma_{r+i j}=\frac{1}{2} \delta_{i j}, \quad \sigma_{i r+j}=\frac{1}{4} \delta_{i j} \quad(i, j=1, \cdots, r) .
$$

Then conditions (64) become identities unless $i>r, k>r$, while then the $\sigma_{i j}$ involved do not occur in (61). Hence we need not consider further the conditions (64).

To make our notations uniform with those at the end of $\S 12$, we set

$$
T=T_{k}, \quad \tau_{i}=-\tau_{i \kappa}, \quad \sigma_{2 r+j, k}=-\frac{1}{2} \beta_{j k \kappa}, \quad \sigma_{i, 2 r+j}=-\frac{1}{4} \alpha_{i k j} .
$$

Then the preceding results give

$$
\begin{gathered}
T_{\kappa}: \delta \xi_{(3 \kappa-2) r+i}=\delta \xi_{(3 \kappa-1) r+i}=\tau_{i \kappa}, \quad \delta \xi_{3 k r+j}=-\sum_{k=1}^{r} \beta_{j k \kappa} \tau_{k \kappa} \quad\left(\begin{array}{l}
i=1, \cdots, r \\
j=1, \cdots, \mu
\end{array}\right), \\
\tau_{i \kappa}=-\frac{1}{2} \xi_{(3 \kappa-2) r+i}-\frac{1}{4} \xi_{(3 \kappa-1) r+i}+\frac{1}{4} \sum_{j=1}^{\mu} \alpha_{i \kappa j} \xi_{3 k r+j} .
\end{gathered}
$$

Since $T_{\kappa}$ must multiply $\tau_{i \kappa}$ by -1 , we have

$$
\sum_{j}^{\mu} \alpha_{i k j} \beta_{j k \kappa}=5 \delta_{k} \quad(i, k=1, \cdots, r) .
$$

Trans. Am. Math. Soc. 10 
The conditions that $T_{\kappa}$ be commutative with $L_{\kappa-1}$ and $L_{t}(t<\kappa-1)$ are

$$
\begin{aligned}
\sum_{j=1}^{\mu} \alpha_{i k j} \beta_{j k k-1} & =\delta_{i k}, & \sum_{j=1}^{\mu} \alpha_{i k-1 j} \beta_{j k \kappa} & =\delta_{i k}, \\
\sum_{j=1}^{\mu} \alpha_{i k j} \beta_{j k t} & =0, & \sum_{j=1}^{\mu} \alpha_{i t j} \beta_{j k k} & =0 .
\end{aligned}
$$

We proceed as in $\S 13$, with an additional value $\kappa$ for $t$. Thus in (55), $A_{i}$ now has an additional row, $B_{k}$ an additional column, while in (56) and (57) there is an additional row and column, the new diagonal element being 5 , the element preceding 5 being 1 , that above 5 being 1 , and the remaining elements being zero, by (68)-(70). Expanding $\left|\pi_{u^{\prime}}\right|$ according to the last row, we get

$$
5 \Delta_{\kappa-1}-\Delta_{\kappa-2}=5 \kappa-(\kappa-1)=4 \kappa+1 .
$$

TheOREM. For $q \geqq 4 \kappa+1$ we have $\mu \geqq \kappa r$ or $\mu \geqq(\kappa-1) r$, according as the field does not have or has a modulus which divides $4 \kappa+1$.

15. Let next $q \geqq 4 \kappa+2$. To $[4 \kappa+1,4 \kappa+2]$ corresponds a transformation commutative with the $\kappa$ triples (35) and (47) and hence not affecting $\xi_{i}(i=1, \cdots, 3 \kappa r)$. Applying a transformation on the remaining variables, we may set

$$
[4 \kappa+1,4 \kappa+2] \sim Q=\prod_{i=1}^{r} C_{3 \kappa r+i} .
$$

Since the the latter must be commutative with $L_{t}$, we have

$$
\alpha_{i j}=0, \quad \beta_{i j t}=0 \quad(i, j=1, \cdots, r ; t=1, \cdots, \kappa-1) .
$$

Since $[4 \kappa, 4 \kappa+1] \sim T_{\kappa}$, given by $(66), T_{\kappa} Q$ must be of period 3 . The necessary and sufficient conditions are

$$
\sum_{j=1}^{r} \alpha_{i k j} \beta_{j k \kappa}=2 \delta_{i k}=\sum_{j=1}^{r} \alpha_{j k k} \beta_{i j k} \quad(i, k=1, \cdots, r) .
$$

Thus $|a| \neq 0$. We make the transformation of variables

$$
\xi_{3 \kappa r+i}^{*}=\frac{1}{2} \sum_{j=1}^{r} \alpha_{i k j} \xi_{3 \kappa r+j}
$$$$
(i=1, \cdots, r) \text {. }
$$

Of the preceding transformations, the only one altered is $T_{\kappa}$. For the latter, $\delta \xi_{3 x+i}=-\tau_{i \kappa}$. The effect of this normalization is the replacement of conditions (73) by

$$
\beta_{j k \kappa}=\delta_{j k}, \quad \alpha_{j k k}=2 \delta_{j k} \quad(j, k=1, \cdots, r) .
$$

The remaining parameters of our transformations define the matrices

$$
A_{i}=\left(\alpha_{i t}\right), \quad B_{i}=\left(\beta_{j i t}\right) \quad(t=1, \cdots, \kappa ; j=r+1, \cdots, \mu ; i=1, \cdots, r) .
$$

These parameters are subject to conditions (52)-(54), (69), (70), in which, by 
(72), we may now take $j=r+1, \cdots, \mu$, and to the conditions

$$
\sum_{j=r+1}^{\mu} \alpha_{i \kappa j} \beta_{j k \kappa}=3 \delta_{i k} \quad(i, k=1, \cdots, r),
$$

which follow from (68) and (74). The statements at the end of $\$ 14$ hold here if we replace 5 by 3 ; in particular, $|\pi|=2 \kappa+1$. In applying the argument of $\S 13$, the two cases to consider are now $\mu-r<\kappa r$ and $\mu-r<(\kappa-1) r$.

Theorem. For $q \geqq 4 \kappa+2$, we have $\mu \geqq(\kappa+1)$ ror $\mu \geqq \kappa r$, according as the field does not have or has a modulus which divides $2 \kappa+1$.

Note that (66) and (67) simplify, in view of (74):

$$
\begin{array}{rrr}
T_{\kappa}: \delta \xi_{(3 \kappa-2) r+i}=\delta \xi_{(3 \kappa-1) r+i}=\tau_{i \kappa}, \quad \delta \xi_{3 \kappa r+i}=-\tau_{i \kappa} & (i=1, \cdots, r)_{2} \\
\delta \xi_{3 \kappa r+j}=-\sum_{k=1}^{r} \beta_{j k \kappa} \tau_{k \kappa} & (j=r+1, \cdots, \mu),
\end{array}
$$

$$
\tau_{i \kappa}=-\frac{1}{2} \xi_{(3 \kappa-2) r+i}-\frac{1}{4} \xi_{(3 \kappa-1) r+i}+\frac{1}{2} \xi_{3 \kappa r+i}+\frac{1}{2} \sum_{j=r+1}^{\mu} \alpha_{i \kappa j} \xi_{3 k r+j} .
$$

16. Finally, let $q=4 \kappa+3$. Since a transformation commutative with the $\kappa$ triples affects only $\xi_{i}(i>3 \kappa r)$, we have

Then, by (71),

$$
[4 \kappa+1,4 \kappa+3] \sim E: \xi_{3 \kappa r+i}^{\prime}=\sum_{j=1}^{\mu} \epsilon_{i j} \xi_{3 k r+j} \quad(i=1, \cdots, \mu) .
$$

$$
[4 \kappa+2,4 \kappa+3] \sim R=E Q E: \delta \xi_{3 \kappa r+i}=-2 \sum_{j=1}^{r} \epsilon_{i j} \omega_{j} \quad(i=1, \cdots, \mu),
$$

in which $\omega_{i}$ denotes the sum (77) for $i \leqq r$. Now $Q$ must transform $R$ into $E$. As in (64), the conditions are

$$
\delta_{i k} \pm 2 \sum_{j=1}^{r} \epsilon_{i j} \epsilon_{j k}=\epsilon_{i k} \quad(i, k=1, \cdots, \mu),
$$

the sign being minus if and only if $i$ and $k$ are both $\leqq r$ or both $>r$. Set

$$
e=\left(\epsilon_{i j}\right), \quad e_{\rho}=e-\rho I
$$$$
(i, j=1, \cdots, r) \text {. }
$$

In view of relations (79) for $i, k=1, \ldots, r$, we have

$$
e_{\rho} e_{\sigma}=\left(\frac{1}{2}+\rho \sigma\right) I+f e \quad\left(f=-\frac{1}{2}-\rho-\sigma\right) .
$$

Set $\Delta_{\rho}=\left|e_{\rho}\right|$. Taking $f=0$; we have

$$
\Delta_{\rho} \Delta_{-1-\rho}=\left[\frac{1}{2}+\rho\left(-\frac{1}{2}-\rho\right)\right]^{r}=(1+\rho)^{r}\left(\frac{1}{2}-\rho\right)^{r} .
$$

We next prove* that $\Delta_{\rho}=0$ actually has the root $\rho=\frac{1}{2}$. For a fixed value of $k(k>r)$, equations $(79)$, with $i=1, \cdots, r$, may be written

$$
\epsilon_{i 1} \epsilon_{1 k}+\cdots+\left(\epsilon_{i i}-\frac{1}{2}\right) \epsilon_{i k}+\cdots+\epsilon_{i r} \epsilon_{r k}=0 .
$$

* This is obvions if the modulus is 3 , since $-1=\frac{1}{2}$. 
For a fixed value of $i(i>r)$, equations (79), with $k=1, \cdots, r$, are

$$
\epsilon_{1 k} \epsilon_{i 1}+\cdots+\left(\epsilon_{k k}-\frac{1}{2}\right) \epsilon_{i k}+\cdots+\epsilon_{r k} \epsilon_{i r}=0 .
$$

Hence, if $\Delta_{i} \neq 0$, then $\epsilon_{i k}=0(i \leqq r, k>r), \epsilon_{i k}=0(i>r, k \leqq r)$, so that $E$ would be commutative with $Q$, and the isomorphism would fail.

It follows that $\Delta_{\rho}=0$ has an $l$-fold root $\frac{1}{2}$ and an $(r-l)$-fold root -1 , where $l \geqq 1$, while $l=r$ if the field has modulus 3. Applying*a transformation on the variables $\xi_{3 k r+i}(i=1, \cdots, r)$, we may, in view of the theory of canonical forms, normalize $e$ and set

$$
\begin{gathered}
\epsilon_{i j}=0(j \neq i, i-1), \quad \epsilon_{i i}=\frac{1}{2}(i=1, \cdots, l), \quad \epsilon_{i i}=-1(i=l+1, \ldots, r), \\
\epsilon_{l+1 l}=0 .
\end{gathered}
$$

Then equations (79), for $i, k=1, \cdots, r$, reduce to

$$
3 \epsilon_{i i-1}=0(i \neq l+1), \quad \epsilon_{i i-1} \epsilon_{i-1 i-2}=0(i=1, \ldots, r)
$$

Let $\kappa>0$. Then $R$ must be commutative with $T_{\kappa}$, given by $\left(66^{\prime}\right)$. By an argument similar to that with $U$ in $\S 14$, the conditions are that

$$
R \text { leaves fixed each } \tau_{i k}, T_{k} \text { each } \omega_{i} \quad(i=1, \cdots, r) .
$$

Suppose first that the field does not have modulus 3. Then by (85), (86), $\epsilon_{i i-1}=0(i=2, \cdots, r)$. Hence (83) and (84) reduce to

$$
\epsilon_{i k}=0(i=l+1, \ldots, r ; k>r), \quad \epsilon_{i k}=0(i>r ; k=l+1, \ldots, r) .
$$

Hence $E$ and $R$ are products of $\Pi C_{3 k r+i}$ by transformations not affecting

$$
\xi_{3 \times r+2} \quad(i=l+1, \cdots, r) .
$$

Then, by $\dagger(87), T_{\kappa}$ leaves unaltered the variables (89). But, for $Q$ defined by (71), $T_{k} Q$ must be of period 3 . Hence $l=r$, and

$$
\left(\epsilon_{i j}\right)=\frac{1}{2} I \quad(i, j=1, \cdots, r) .
$$

Next let the field have modulus 3. Then $\epsilon_{i i}=-1(i \leqq r)$. Suppose that $\epsilon_{i i-1} \neq 0$ for a fixed value of $i, 2 \leqq i \leqq r$. Then by (86) and (83),

$$
\epsilon_{i-1 ;-2}=0, \quad \epsilon_{i-1 k}=0 \quad(k=r+1, \cdots, \mu) .
$$

Then $\omega_{i-1}=-\xi_{3 \times r+i-1}$; so that, by (87), $T_{\kappa}$ leaves $\xi_{3 \times r+i-1}$ unaltered. Then $T_{k} Q$ is not of period 3, contrary to the isomorphism. Hence each $\epsilon_{i i-1}=0(i \leqq r)$, and relation (90) holds.

- Having established (90), we introduce notations uniform with those of the preceding sections. We set

$$
R=T_{\kappa+1}, \quad \omega_{i}=-\tau_{i k+1}, \quad \epsilon_{j i}=-\frac{1}{2} \beta_{j i k+1}, \quad \epsilon_{i j}=-\frac{1}{4} \alpha_{i \kappa+1} \quad(i \leqq r ; j>r) .
$$

* If $\kappa>0$, wo forego for the present the normalization (74) of $T_{k}$.

†To give a direct proof, we note that $T_{k}$ multiplies each variable (89) by \pm 1 , and that $\pm 1=+1$ by the hypothesis in 811 . 
Then by (78), (90), and the definition of $\omega_{i}$ às the sum (77),

(91) $T_{k+1}: \delta \xi_{3 k r+i}=\tau_{i k+1}, \delta \xi_{3 k+j}=-\sum_{k=1}^{r} \beta_{j k k+1} \tau_{k k+1}(i=1, \cdots, r ; j=r+1, \cdots ; \mu)$ :

$$
\tau_{i x+1}=-\frac{1}{2} \xi_{3 x+i}+\frac{1}{j} \sum_{j=r+1}^{\mu} a_{i k+1} \xi_{3 \times r+j}
$$

In view of its origin, $T_{\kappa+1}$ must multiply $\tau_{i \kappa+1}$ by -1 , whence

$$
\sum_{j=r+1}^{\mu} \alpha_{i k+1 j} \beta_{j k x+1}=6 \delta_{i k} \quad(i, k=1, \cdots, r) .
$$

Without altering (90) we may make the normalization in $\S 15$, by means of which $T_{k}$ takes the form $\left(66^{\prime}\right)$. In view of $(87), T_{k}$ and $T_{k+1}$ are commutative if and only if

$$
\sum_{j=r+1}^{\mu} \alpha_{i k+1 j} \beta_{j k k}=2 \delta_{i k}, \quad \sum_{j=r+1}^{\infty} \alpha_{i k j} \beta_{j k k+1}=2 \delta_{i k} \quad(i, k=1, \cdots, r) .
$$

Finally, $T_{k+1}$ is commutative with $L_{\imath}$, given by (50), (51), (72), if and only if

$$
\sum_{j=r+1}^{\mu} \alpha_{i \kappa+1 j} \beta_{j k t}=0, \sum_{j=r+1}^{\mu} \alpha_{i t j} \beta_{j k \kappa+1}=0 \quad(i, k=1, \cdots, r ; t=1, \cdots, \kappa-1) .
$$

We proceed as at the end of $\$ 15$. We consider matrices (75) for $t=1, \ldots, \kappa+1$, viz., with an additional row in $A_{i}$ and an additional column in $B_{i}$, the new elements satisfying (93)-(95). Thus, for $i \neq k, A_{i} B_{k}$ is a matrix all of whose elements are zero; while

$$
A_{i} B_{i}=\left(\begin{array}{cccccccccc}
2 & 1 & 0 & 0 & \ldots & 0 & 0 & 0 & 0 & 0 \\
1 & 2 & 1 & 0 & \ldots & 0 & 0 & 0 & 0 & 0 \\
\cdot & . & . & . & . & . & & . & . & . \\
0 & 0 & 0 & 0 & \ldots & 0 & 1 & 2 & 1 & 0 \\
0 & 0 & 0 & 0 & \ldots & 0 & 0 & 1 & 3 & 2 \\
0 & 0 & 0 & 0 & \ldots & 0 & 0 & 0 & 2 & 6
\end{array}\right),
$$

a square matrix of order $\kappa+1$, whose determinant equals $(\$ \S 13,15)$

$$
6(2 \kappa+1)-2^{2} \Delta_{\kappa-1}=2(4 \kappa+3) .
$$

Considering as in $\S 13$ the cases $\mu-r<(\kappa+1) r$ and $\mu-r<\kappa r$, we obtain the following

TheоRiм. For $q=4 \kappa+3$, we have $\mu \geqq(\kappa+2) r$ or $\mu \geqq(\kappa+1) r$, according as the field does not have or has a modulus which divides $q$.

17. Under the hypothesis made in $\$ 11$ that $8=0$, we have exhibited in \$\$11-16 the correspondence of generators in any simple isomorphism betewno 
the symmetric group on $q$ letters and an $m$-ary linear homogeneous group in a field not having modulus 2 , and have obtained lower limits on $\mu \equiv m-3 \kappa r$. The latter results may be combined into the following

Theorem. The minimum value of $m$ is $(q-1) r$ or $(q-2) r$, according as the field does not have or has a modulus which divides $q$.

For $r=1$, the hypothesis $s=0$ is satisfied and we have the

CoRollary. In any representation of the symmetric group on $q$ letters as an m-ary linear homogeneous group, such that a transposition corresponds to a transformation conjugate with $C_{1}$, it is necessary that $m \geqq q-2$, while $m=q-2$ only when the field has a modulus which divides $q$.

18. Principle of duality. If the general $m$-ary linear homogeneous group $G L H\left(m, F^{\prime}\right)$ in a field $F$, not having modulus 2 , contains a subgroup $G_{q}^{(r)}$ ? of symmetric type, such that a transposition corresponds to a transformation conjugate with $C_{1} \ldots C_{r}$, then $G L H(m, F)$ contains a subgroup $G_{q !}^{(m-r)}$.

As this is obviously true when $q=2$, we set $q>2$. Then there is no invariant substitution, so that $C=C_{1} \ldots C_{m}$ cannot occur in $G_{q !}^{(r)}$. In particular, $r<m$.

Let the even substitutions correspond to the transformations $e_{1}, e_{2}, \ldots$ of $G_{q !}^{(r)}$, and the odd substitutions to $o_{1}, o_{2}, \ldots$ Then

$$
e_{1}, e_{2}, \cdots, o_{1} C, o_{2} C, \cdots
$$

are all distinct and form an isomorphic group $G_{q !}^{(m-r)}$.

19. Lемма. The symmetric group on $m+3$ letters cannot be represented as an m-ary linear homogeneous group in a field not having modulus 2.

The proof proceeds by induction from $m-2$ to $m$, the lemma being evidently true for $m=1$, and true for $m=2$ by $\S 17$, since then $r=1$ by $\S 18$.

Suppose that there is a representation $G_{(m+3) !}^{(r)}$. By $\$ \S 17,18$ we may set

$$
2 \leqq r \leqq \frac{1}{2} m, \quad[12] \sim C_{1} \ldots C_{r} .
$$

Any transformation commutative with $C_{1} \ldots C_{r}$ is necessarily a product $S_{1, \ldots, r} S_{r+1, \ldots, m}^{\prime}$. Hence by $\S 7$ the symmetric group on the $m+1$ letters $3, \ldots, m+3$ is simply isomorphic with a linear homogeneous group on $r$ or $m-r$ variables. By $\left(97_{1}\right), r \leqq m-2, m-r \leqq m-2$. Hence the hypothesis for the induction is contradicted.

20. Suppose, finally, that the symmetric group on $m+2$ letters is representable as an $m$-ary linear homogeneous group in a field $F$ not having modulus 2 . If $r=1$ or $m-1, F$ must have a modułus which divides $m+2(\S 17)$. If $1<r<m-1$, we may assume that (97) holds. As in $\S 19$, the symmetric group $G$ on the $m$ letters $3, \ldots, m+2$ is simply isomorphic with a group on $r$ 
or else $m-r$ variables; while by the lemma there proved the number of variables is $\geqq m-2$. For either alternative, it follows from $\left(97_{1}\right)$ that $r=2$. Then $m \geqq 4$, so that ( $\S 4$ or $\S \S 17-19) G$ cannot be represented on $r=2$ variables.

Hence by the proof given in $\S 7$, there remain only two possibilities :

(i) The group $G$ affects only the variables $\xi_{3}, \ldots, \xi_{m}$;

(ii) The transformations of $G$ corresponding to even substitutions affect only the variables $\xi_{3}, \ldots, \xi_{m}$; while those corresponding to odd substitutions are products of transformations on $\xi_{3}, \cdots, \xi_{m}$ by a fixed transformation $T$, of period 2 , on $\xi_{1}$ and $\xi_{2}$.

For case (i), we have (18) with $r=2, s=0$. Hence by $\S 17$, the minimum number of variables in a representation of $S_{(m+2) !}$ is $2 m$. We may proced directly and conclude from $[12] \sim C_{1} C_{2},[34] \sim C_{3} C_{4},[56] \sim C_{5} C_{6}, \ldots$ that at least $m+2$ or $m+1$ variables are necessary, according as $m+2$ is even or odd.

For case (ii), we apply a transformation on $\xi_{1}, \xi_{2}$, and set $T=C_{1}$, since obviously. $T \neq C_{1}^{\gamma} C_{2}$. Applying a transformation on $\xi_{3}, \cdots, \xi_{m}$, we may set [34] $\dot{C}_{1} C_{3},[56] \sim C_{1} C_{4}$, and; as before, [12] $C_{1} C_{2}$. The case thus falls under $\S 9$ with $r=2, s=1$. Then by $(20)$ we may set [14] [23] $\left(\xi_{2} \xi_{3}\right) C_{2} C_{3} C_{k}$, where $k=1$ if $\sigma=1, k=4$ if $\sigma=0$. Let [23] W. Since [23] is commutative with [56] and [14][23], we have $W=(a)_{1}(b)_{4} S, S$ affecting only $\xi_{2}, \xi_{3}, \xi_{5}, \ldots$. Since [12] [23] is of period 3, we have $a=-1$. If $b=-1,[23]$ and $[56]$ both correspond to $C_{1} C_{4}$. Hence $W=C_{1} S$. It follows that $S_{(m+2)}$ is represented as a subgroup of the direct product of $\left\{I, C_{1}\right\}$ and a group on $\xi_{2}, \cdots, \xi_{m}$, and hence $(\S 7)$ is simply isomorphic with a group on $m-1$ variables. But this contradicts $\S 19$.

Hence cases (i) and (ii) are both excluded.

TheоREм. If the symmetric group on $q$ letters is representable as a linear homogeneous group on $q-2$ variables in a field $F$ not having modulus 2, then $r=1$ or $q-3$, and $F$ has $a$ modulus which divides $q$.

\section{In view of $\S \S 8,19,20$, we may state the complete}

TheOREM. Let $F$ be an arbitrary field. Then the minimum number of variables for the representation of the symmetric group on $q$ letters is $q-1$ or $q-2$, according as $F$ has not or has a modulus which divides $q$. In the latter case exceptions arise for $q \leqq 4$; when the modulus divides $q$, the minimum number of variables is 2 for $q=2$ or 3 , and 3 for $q=4$.

22. In order to obtain a clear insight into the nature of the linear groups exhibited in $\$ \S 11-16$, we shall make a normalization by a transformation of variables which does not disturb the correspondences (35) and (47). To normalize the $L_{t}$, given by (50), set

$$
\eta_{3 k r+i}=\sum_{j=1}^{\mu} \lambda_{i j} \xi_{3 k r+j} \quad(i=1, \cdots, \mu) .
$$


Then, under $L_{t}$,

$$
\delta \eta_{3 k r+i}=-\sum_{k=1}^{r} \beta_{i k t}^{\prime} \tau_{k t}, \quad \beta_{i k t}^{\prime}=\sum_{j=1}^{\mu} \lambda_{i j} \beta_{j k t} .
$$

Suppose first that the field does not have a modulus which divides $\kappa$. Then, by $\S 13, \mu \geqq(\kappa-1) r . \quad$ As in (55) and (58), set

$$
B_{k}^{\prime}=\left(\beta_{j k t}^{\prime}\right), \quad B^{\prime}=\overbrace{B_{1}^{\prime} \cdots B_{.}^{\prime}}^{\prime} \quad(t=1, \cdots, \kappa-1 ; j=1, \cdots, \mu) .
$$

Thus $(\lambda) B=B^{\prime}$. Now $|A B|$ equals the sum of all products of the determinants of order $(\kappa-1) r$ formed from $(\kappa-1) r$ columns of $A$ by the corresponding determinants formed from $(\kappa-1) r$ rows of $B$. But $|A B| \neq 0$ by (59). Hence, after making a suitable permutation of the variables $\xi_{3 \kappa r+j}$, we may set $|\bar{B}| \neq 0$, where

$$
\bar{B}=\overbrace{\bar{B}_{1} \cdots \bar{B}_{r}}, \quad \bar{B}_{k}=\left(\beta_{j k t}\right) \quad[t=1, \cdots, \kappa-1 ; j=1, \cdots,(\kappa-1) r],
$$

so that $\bar{B}_{k}$ is composed of the first $(\kappa-1) r$ rows of $B_{k}$. For the purpose of assigning an order to the $\beta$ 's in the $j$ th row of $\bar{B}$, we set

$$
\beta_{j k t} \equiv \beta_{j,(k-1)(k-1)+t} \text {. }
$$

For a fixed value $\leqq \mu$ of $i$, the equations

$$
\beta_{i k t}^{\prime}=\delta_{i,(\kappa-1)(k-1)+t} \quad(t=1, \cdots, \kappa-1 ; k=1, \cdots, r)
$$

uniquely determine $\lambda_{i 1}, \ldots, \lambda_{i,(k-1) r}$ as linear functions of the remaining $\lambda_{i j}$, since the determinant of the coefficients of the former equals $|\bar{B}|$. Let $(\lambda)$ denote the matrix in (98) and

$$
L=\left(\lambda_{i j}\right) \quad[i=1, \cdots, \mu ; j=(\kappa-1) r+1, \cdots, \mu] .
$$

We have the following relation between matrices of order $\mu$ :

$$
(\lambda)(\overbrace{B \Omega})=(\overbrace{B^{\prime} L}),
$$

where $\Omega$ is a matrix of $\mu$ rows and $\mu-(\kappa-1) r$ columns, all of whose elements are zero,except those lying in the main diagonal of $\widetilde{B \Omega}$ which equal unity, i. e.,

$$
\Omega=\left(\delta_{i,(\kappa-1) r+j}\right) \quad[i=1, \cdots, \mu ; j=1, \cdots, \mu-(\kappa-1) r] .
$$

Hence by (103) and (105),

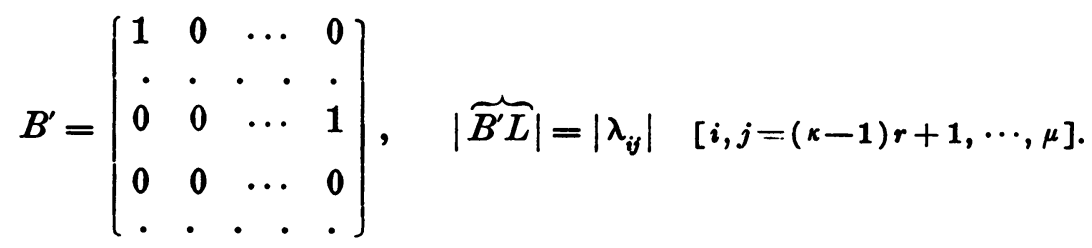

Hence, by (105), if we take the last determinant different from zero, we have $|\lambda| \neq 0$ in (98). 
We assume that this preliminary normalization has been made, so that in $L_{t}$ matrix $B$ has the form (107,). Let

$$
\left.A_{i}=\left(\alpha_{i t j}\right), \quad \bar{A}=\begin{array}{c}
\bar{A}_{1} \\
\vdots \\
\bar{A}_{r}
\end{array}\right\} \quad[t=1, \cdots, \kappa-1 ; j=1, \cdots,(\kappa-1) r],
$$

so that $A_{i}$ is composed of the first $(\kappa-1) r$ columns of $A_{i}$. Then $\bar{A}=\bar{A} B=A B$, so that $\bar{A}=M$, where $M$ is the matrix displayed in (59). Set

$$
a_{i}=(\kappa-1)(i-1) \quad(i=1, \cdots, v) .
$$

Then the final equations in (50) may be replaced by

$$
\delta \xi_{3 k r+a_{i}+t}=-\tau_{i t} \quad(i=1, \cdots, r) .
$$

In (51) the terms of the sum given by $j=1, \ldots,(\kappa-1) r$ reduce to

$$
\frac{1}{4}\left(\xi_{3 \kappa r+a_{i}+t-1}+2 \xi_{3 \kappa r+a_{i}+t}+\xi_{3 \kappa r+a_{i}+t+1}\right),
$$

subject to the restrictions noted on (113). Apply the transformation of variables

$$
\eta_{3 \kappa r+i}=\xi_{3 \kappa r+i}+\sum_{j=(\kappa-1) r+1}^{\mu} \lambda_{i j} \xi_{3 \kappa r+j} \quad[i=1, \cdots,(\kappa-1) r],
$$

from which $\xi=\eta-\sum \lambda \eta$. Then $L_{t}$ is altered in form and

$$
\begin{aligned}
\tau_{i t}(\xi) & =\tau_{i t}(\eta)-\frac{1}{4} \sum_{j=(\kappa-1) r+1}^{\mu} \alpha_{i t j}^{\prime} \eta_{3 \kappa r+j}, \\
\alpha_{i t j}^{\prime} & \equiv \lambda_{a_{i}+t-1, j}+2 \lambda_{a_{i}+t, j}+\lambda_{a_{i}+t+1, j} .
\end{aligned}
$$

Hence $\left(\alpha^{\prime}\right)=M\left(\lambda_{i j}\right)$, where $\left(\lambda_{i j}\right)$ is the matrix in (110). Taking $(\lambda)=M^{-1}(\alpha)$, we have $\left(\alpha^{\prime}\right)=(\alpha)$. Hence the effect of this normalization is to set $\alpha_{i, j}=0$ for $j>(\kappa-1) r$. Finally, we replace

$$
\xi_{3 \kappa r+a_{i}+t} \text { by } \xi_{3 \kappa r+(t-1) r+i} \quad(i=1, \cdots, r ; t=1, \cdots, \kappa-1),
$$

noting that $a_{i}+t=a_{k}+t^{\prime}$ implies $i=k, t=t^{\prime}$. Hence

$$
L_{t} \text { : first four equations as in }(50), \delta \xi_{(3 \kappa+t-1) r+i}=-\tau_{i t} \quad(i=1, \cdots, r),
$$

where $\tau_{i t}$ is given by (51) with the final sum replaced by

$$
\frac{1}{4}\left(\xi_{(3 \kappa+t-2) r+i}+2 \xi_{(3 \kappa+t-1) r+i}+\xi_{(3 \kappa+t) r+i}\right),
$$

the first term being absent if $t=1$, the third if $t=\kappa-1$. We have now proved, for $q=4 \kappa$, the following

THEOREM. For any field $F$ having neither modulus 2 nor a modulus which divides $q$, all linear homogeneous groups in $F$, which are simply isomorphic with the symmetric group on $q$ letters and which satisfy the condition 
$s=0$ imposed in $\S 11$, are equivalent under linear transformation in $F$ to a direct product of $r$ linear groups, with fixed coefficients cogredient in the $r$ sets of variables

$$
\xi_{i}, \xi_{r+i}, \xi_{2 r+i}, \cdots, \xi_{(q-2) r+i} \quad(i=1, \cdots, r) .
$$

To prove the theorem for the remaining cases, we normalize $T_{k}$ and $T_{k+1}$ simultaneously with the $L_{t}$.

First, let $q=4 \kappa+1$. For $T_{\kappa}$, given by (66), we have (99) with $t=\kappa$. The above proof is to be modified by changing $\kappa-1$ to $\kappa$, allowing $t$ to take the additional value $\kappa$, and by taking as $M$ the matrix defined at the end of $\S 14$. We find that $L_{t}$ is given by (112), with no suppression in $\tau_{i t}$ when $t=\kappa-1$, and that

$$
\begin{gathered}
T_{k}: \delta \xi_{(3 k-2) r+i}=\delta \xi_{(3 k-1) r+i}=\tau_{i k}, \quad \delta \xi_{(4 k-1) r+i}=-\tau_{i k} \quad(i=1, \cdots, r), \\
\tau_{i k}=-\frac{1}{2} \xi_{(3 k-2) r+i}-\frac{1}{4} \xi_{(3 k-1) r+i}+\frac{1}{4} \xi_{(4 k-2) r+i}+\frac{5}{4} \xi_{(4 k-1) r+i} .
\end{gathered}
$$

For $q=4 \kappa+2$, we apply transformation (99) with $i, j=r+1, \cdots, \mu$, and modify the proof in the light of $\S 15$. In particular, $t=1, \cdots, \kappa$, and $j>r$ in (100), (101). Hence

$$
L_{t}: \text { first four equations as in }(50),
$$$$
\delta \xi_{(3 x+t) r+i}=-\tau_{\dot{u}} \quad(i=1, \cdots, r),
$$
where $\tau_{i t}$ is given by $(51)$ with the final sum replaced by

$$
\frac{1}{4}\left(\xi_{(3 k+t-1) r+i}+2 \xi_{(3 k+t) r+i}+\xi_{(3 k+t+1) r+i}\right),
$$

the first term being absent if $t=1$. Furthermore,

(119) $T_{\kappa}$ : first three equations as in $\left(66^{\prime}\right), \quad \delta \xi_{4 k r+i}=-\tau_{i \kappa} \quad(i=1, \cdots, r)$,

(120) $\tau_{i k}=-\frac{1}{2} \xi_{(3 k-2) r+i}-1 \frac{1}{4(3 k-1) r+i}+\frac{1}{2} \xi_{3 k r+i}+1 \xi_{(4 k-1) r+i}+\frac{3}{4} \xi_{4 k r+i} \cdot$

Finally, for $q=4 \kappa+3$, the proof is quite similar to that in the preceding case. Here $t$ takes the further value $\kappa+1$. We obtain the same normalized forms for $L_{\tau}$ and $T_{\kappa}$, except that now

Furthermore,

$$
\tau_{i \kappa}=\frac{1}{2} \xi_{(4 k+1) r+i}+\text { the five terms of }(120) \text {. }
$$

$$
\begin{gathered}
T_{\kappa+1}: \delta \xi_{3 \kappa r+i}=\tau_{i \kappa+1}, \quad \delta \xi_{(4 \kappa+1) r+i}=-\tau_{i \kappa+1} \quad(i=1, \cdots, r), \\
\tau_{i \kappa+1}=-\frac{1}{2} \xi_{3 \kappa r+i}+\frac{1}{2} \xi_{4 k r+i}+\frac{8}{2} \xi_{(4 \kappa+1) r+i} .
\end{gathered}
$$

23. Since the linear groups exhibited in $\$ 22$ are direct products of $r$ similar groups, it suffices to know the character of the component groups. We shall list the quadratic invariants of the groups for the case $r=1$, assuming still that the field has neither modulus 2 , nor a modulus dividing $q$. Set

$$
f=\xi_{1}^{2}+\xi_{2}^{2}+\frac{1}{2} \xi_{3}^{2}+\xi_{4}^{2}+\xi_{5}^{2}+\frac{1}{2} \xi_{6}^{2}+\cdots+\xi_{3 k-2}^{2}+\xi_{3 k-1}^{2}+\frac{1}{2} \xi_{3 k}^{2} \cdot
$$


Then the only quadratic invariant of the group is

$$
\begin{aligned}
& f+\xi_{3 k+1}^{2}+\xi_{3 k+1} \xi_{3 k+2}+\xi_{3 k+2}^{2}+\xi_{3 k+2} \xi_{3 k+3}+\xi_{3 k+3}^{2}+\cdots \\
& +\xi_{4 k-2}^{2}+\xi_{4 k-2} \xi_{4 k-1}+\xi_{4 k-1}^{2} \\
& (q=4 \kappa), \\
& \text { terms of }(125)+\xi_{4 k-1} \xi_{4 k}+\frac{5}{2} \xi_{4 k} \\
& (q=4 \kappa+1), \\
& f+\xi_{3 k+1}^{2}+\xi_{3 k+2}^{2}+\xi_{3 k+2} \xi_{3 \kappa+3}+\xi_{3 k+3}^{2}+\cdots \\
& +\xi_{4 \kappa-1}+\xi_{4 \kappa-1} \xi_{4 \kappa}+\xi_{4 \kappa}^{2}+\xi_{4 \kappa} \xi_{4 \kappa+1}+\frac{3}{2} \xi_{4 \kappa+1}^{2} \\
& (q=4 \kappa+2), \\
& \text { terms of }(127)+2 \xi_{4 k+1} \xi_{4 k+2}+3 \xi_{4 k+2}^{2} \\
& (q=4 \kappa+3) .
\end{aligned}
$$

The discriminant of the quadratic invariant is $2^{l}|P|$, where $l=2 \kappa$ in the first two cases and $l=2 \kappa+1$ in the last two ; while, for $q=4 \kappa, P$ is the matrix (57) of order $\kappa-1$; for $q=4 \kappa+1$ and $4 \kappa+2, P$ is the matrix of order $\kappa$ defined at the end of $\S \S 14$ and 15 ; and for $q=4 \kappa+3, P$ is the matrix (96) of order $\kappa+1$. The matrix of the discriminant therefore reflects an essential property of the group. In each case the discriminant is not zero.

24. In conclusion, we consider briefly the normalization of our linear groups for the case of fields having a modulus $p(p>2)$ which divides $q$. It is no longer true that, for a given value of $r$, the groups are all equivalent under linear transformation. For simplicity, we consider only the case in which the number of variables is the minimum $(q-2) r$.

Let first $q=4 \kappa$. Then by hypothesis $\mu=(\kappa-2) r$, and the modulus divides $\kappa$. Matrices $A^{*}$ and $B^{*}$, defined at the end of $\S 13$, are now square matrices of order $\mu=(\kappa-2) r$, and each has its determinant $\neq 0$ by $\left(59^{*}\right)$. Hence in (98) and (99) we may take $\left(\lambda_{i j}\right)=\left(B^{*}\right)^{-1}$. After this normalization, we have $B^{*}=I$. Now by (59),

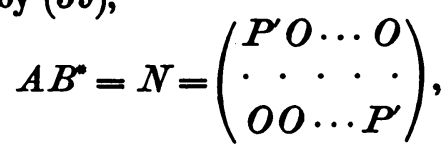

where $P^{\prime}$ is derived from $P$, given by (57), by deleting the last column. Hence $A=N$. The $\beta_{j k_{k-1}}$ are now uniquely determined by (59). Set

Then

$$
\beta_{(\kappa-2)(i-1)+v, k, \kappa-1}=b v \quad(i, k=1, \cdots, r ; v=1, \cdots, \kappa-2) .
$$

$$
\begin{gathered}
2 b_{1}+b_{2}=0, \quad b_{1}+2 b_{2}+b_{3}=0, \ldots, \quad b_{v-1}+2 b_{v}+b_{v+1}=0, \cdots, \\
b_{k-3}+2 b_{k-2}=\delta_{i k}, \quad b_{k-2}=2 \delta_{i k} .
\end{gathered}
$$

Since the eliminant of these $x-1$ equations is $|P|$, a multiple of the modulus, there is a unique set of solutions :

$$
b_{v}=(-1)^{\kappa-v}(\kappa-v) \delta_{i k} .
$$


Proceeding similarly when $q=4 \kappa+1, \ldots, 4 \kappa+3$, we obtain the

Theorem. When the field $F$ has a modulus $p(p>2)$ which divides $q$, all linear homogeneous groups, which are simply isoncorphic with the symmetric groups on $q$ letters, and which affect the minimum number $(q-2) r$ of variables under the hypothesis of $\$ 11$, are equivalent under linear transformation in $F$ to a direct product of $r$ cogredient linear groups with fixed coefficients.

It is sufficient to exhibit one of the component groups. Hence we set $r=1$.

For $q=4 \kappa, L_{t}(t \leqq \kappa-2)$ is defined by (112) for $r=i=1$, with the first term of (113) deleted if $t=1$, and the last term if $t=\kappa-2$. Also

$$
\begin{gathered}
L_{k-1}: \delta \xi_{3 k-4}=\delta \xi_{3 k-3}=\tau_{k-1}, \quad \delta \xi_{3 k-2}=\delta \xi_{3 k}=-\tau_{k-1}, \quad \delta \xi_{3 k+j}=(-1)^{k-j} j \tau_{k-1} \\
(j=1, \cdots, \kappa-2), \\
\tau_{k-1}=-\frac{1}{2} \xi_{3 k-4}-\frac{1}{4} \xi_{3 k-3}+\frac{1}{2} \xi_{3 k-2}+\frac{1}{4} \xi_{3 k}+1 \xi_{4 k-2} .
\end{gathered}
$$

For $q=4 \kappa+1, L_{t}$ is given by (112) for $r=i=1$, with the suppressions noted on (113), while

$$
\begin{aligned}
T_{\kappa}: \quad \delta \xi_{3 \kappa-1}=\delta \xi_{3 \kappa} & =\tau_{\kappa}, \quad \delta \xi_{3 \kappa+j}=-(-1)^{\kappa-j} 4 j \tau_{\kappa} \quad(j=1, \cdots, \kappa-1), \\
\tau_{k} & =-\frac{1}{2} \xi_{3 k-1}-\frac{1}{4} \xi_{3 \kappa}+1 \xi_{4 k-1} .
\end{aligned}
$$

For $q=4 \kappa+2, L_{t}$ is given by (117) for $r=i=1$, with the first term of (118) suppressed if $t=1$, and the last term if $t=\kappa-1$. Also

(133) $T_{k}: \delta \xi_{3 k-1}=\delta \xi_{3 k}=\tau_{k}, \delta \xi_{3 k+1}=-\tau_{k}, \delta \xi_{3 k+j}=(-1)^{k-j}(2 j-2) \tau_{k}(j=2, \cdots, k)$,

$$
\tau_{\kappa}=-\frac{1}{2} \xi_{3 \kappa-1}-1 \xi_{3 k}+\frac{1}{2} \xi_{3 \kappa+1}+1 \xi_{4 \kappa} \text {. }
$$

Finally, for $q=4 \kappa+3, L_{t}$ is given by (117), with the first term of (118) suppressed if $t=1 ; T_{\kappa}$ is given by $(119)$, while

(135) $T_{\kappa+1}: \delta \xi_{3 k+1}=\tau_{\kappa+1}, \quad \delta \xi_{2 k+j}=-(-1)^{\kappa-j}(4 j-4) \tau_{\kappa+1}(j=2, \cdots, \kappa+1)$,

$$
\tau_{\kappa+1}=-\frac{1}{2} \xi_{3 k+1}+\frac{1}{2} \xi_{4 k+1} \text {. }
$$

We may now show that in the present case for which the modulus divides $q$, the quadratic invariant of the group is obtained from the invariant when the modulus is prime to $q$ by suppressing the final two terms. For instance, if $q=4 \kappa+1$ the invariant is (125), if $q=4 \kappa+3$ the invariant is (127).

The Univeresity of Chicago, $M \sim a h, 1907$. 